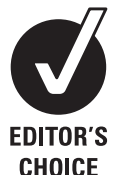

${ }^{1}$ Royal Hallamshire Hospital, Sheffield Teaching Hospitals NHS Trust, Sheffield, South Yorkshire, UK

${ }^{2}$ Barnsley Hospital NHS Foundation Trust, Barnsley, South Yorkshire, UK

\section{Correspondence to} Dr R E Musson, Department of Radiology, C Floor, Royal Hallamshire Hospital, Glossop Rd, Sheffield S10 2JF, UK; rachel.musson@sth.nhs.uk

Received 1 December 2009 Accepted 7 December 2010 Published Online First 17 January 2011

\title{
Gas patterns on plain abdominal radiographs: a pictorial review
}

\author{
Rachel E Musson, ${ }^{1}$ lan Bickle, ${ }^{1}$ Ram K P Vijay ${ }^{2}$
}

\begin{abstract}
Abdominal radiographs are one of the most commonly performed radiological examinations and have an established role in the assessment of the acute abdomen. The main indication is for suspected bowel obstruction and in conjunction with an erect chest x-ray for suspected visceral perforation. Often, the pattern of gas points to a particular pathology, and accurate interpretation is important for prompt diagnosis. The diagnosis in most cases will be confirmed by further imaging studies such as ultrasound, contrast studies or, most commonly in contemporary practice, CT. This pictorial review summarises the various types of intraluminal and extraluminal gas patterns, illustrates some of the common clinical diagnoses made from plain films, describes some commonly encountered clinical problems with radiographs, and discusses the role of advanced imaging techniques.
\end{abstract}

\section{INTRODUCTION}

Abdominal radiographs have an important, wellestablished role in the initial evaluation of the acute abdomen in the emergency setting. It remains one of the most commonly requested investigations despite advances in other imaging modalities, such as ultrasound, CT and MRI, and regardless of the limited evidence supporting their use.

Although plain radiographs generally have a low diagnostic yield and the majority show a nonspecific pattern of gas distribution, there are several patterns that may point towards a particular diagnosis and hence influence further management. The patterns distinctive of particular abnormalities are often subtle and may be overlooked if not considered when evaluating the plain radiograph.

Our aim in this pictorial review article is to describe the assessment of the plain film and the normal patterns of expected air and to examine the distribution of abnormal gas pattern, divided into intraluminal and extraluminal groups. We will (a) illustrate the range of diagnoses that can be made from the plain radiograph, thus guiding further management, particularly the judicious and timely use of CT imaging, (b) discuss commonly encountered clinical problems related to abdominal radiograph imaging, and $(c)$ describe the role of more advanced imaging techniques.

\section{INTERPRETATION OF THE PLAIN ABDOMINAL FILIM}

Interpretation of plain abdominal radiograph involves an assessment of the gas, fluid, soft tissue, fat and calcific densities. ${ }^{1-3}$ This review concentrates specifically on the analysis of patterns of gas. Normal gas in the abdomen is predominantly due to swallowed air.

Air in the stomach is often seen, the pattern being somewhat variable with air fluid levels being commonly present. Small bowel air usually appears as multiple, small, randomly distributed, gaseous foci scattered throughout the abdomen. Small bowel gas is increased in patients who chronically swallow air or drink carbonated beverages. A normal small bowel gas pattern varies from no gas being visible to gas in three or four variably shaped small intestinal loops.

It is usually possible to differentiate between dilated small and large bowel on a plain abdominal radiograph. Clues include the distribution of bowel loops - the small bowel is usually located centrally and the large bowel is usually peripheral. This is referred to as 'picture in a frame'-the small bowel being the 'picture' in the centre and the large bowel the 'frame' around the edge. The fold pattern also differentiates between small and large bowel. The small bowel folds are called valvulae conniventes, which extend across the entire lumen of the bowel loop, and the large bowel folds are haustra, which only span part way across the lumen (usually about one-third). The large bowel often has a mottled appearance in the lumen, which is representative of faecal residue.

The small bowel is dilated when its transverse diameter exceeds $25 \mathrm{~mm}$ in the more distal ileum and $30 \mathrm{~mm}$ more proximally in the jejunum. Air fluid levels in the small bowel are common but should not exceed $25 \mathrm{~mm}$ in length. The diameter of the colon at the caecum should not exceed $80 \mathrm{~mm}$, and the remainder of the colon should not exceed a diameter of $55 \mathrm{~mm}$. Air fluid levels should not be seen distal to the hepatic flexure.

\section{TYPES OF GAS PATTERNS}

Gas patterns can be broadly categorised into intraluminal or extraluminal on the basis of the anatomical location of the normal or abnormal gas within or outside the bowel (table 1).

\section{Intraluminal}

Analysis of the intraluminal gas pattern involves examining gas patterns in the lumen of the stomach, bowel and other luminal structures, such as the gallbladder, portal vein and urinary bladder, and recognising displacement of the normal bowel gas distribution due to intra-abdominal pathology.

\section{Extraluminal}

Gas seen outside the bowel lumen is abnormal, the most common site being the intraperitoneal cavity. 
Table 1 Classification of bowel gas patterns

\begin{tabular}{ll}
\hline Intraluminal & Extraluminal \\
\hline 1. Gas within bowel lumen & 1. Free intraperitoneal gas \\
2. Gas within other luminal & 2. Gas within the wall (intramural) \\
structures & of a viscus \\
3. Bowel gas displacement & 3. Gas within a collection or abscess \\
& 4. Gas within the abdominal wall \\
\hline
\end{tabular}

Certain signs are recognised on plain films, the most well cited being free gas under the diaphragm and Rigler's sign. Free air in the retroperitoneum shows a different pattern of distribution. Gas can also be seen in the wall of a viscus (intramural air; indicative of particular pathologies), in an abscess or collection deep in the abdomen, or in the abdominal wall.

\section{DIAGNOSES FROM ABDOMINAL RADIOGRAPHS}

Many different diagnoses can be made from plain films. We have divided these into intraluminal and extraluminal patterns and show a number of different examples.

\section{Gas within the alimentary tract}

Small bowel obstruction

Small bowel obstruction is a common surgical problem and presents with a variable degree of abdominal pain, vomiting and abdominal distension. Plain radiography is commonly performed in the acute setting in the accident and emergency department or surgical assessment wards to look for specific evidence to support this diagnosis.

The findings on plain film are dilated loops of small bowel, measuring over $30 \mathrm{~mm}$ proximally and $25 \mathrm{~mm}$ more distally. Air fluid levels exceeding $25 \mathrm{~mm}$ in length are abnormal, and air fluid levels at different positions within the same loop-the 'step ladder' pattern-and small bubbles trapped between dilated loops-the 'string of pearls sign'-are also indicative of small bowel obstruction. Less commonly, if the small bowel loops are dilated but completely filled with fluid, the plain $\mathrm{x}$-ray will show a gasless abdomen, which should raise the suspicion of obstruction in patients in whom it is suspected clinically. The radiographic findings may be apparent $6-12 \mathrm{~h}$ before the clinical symptoms. Figure 1 shows some of the radiographic findings of small bowel obstruction.

Small bowel obstruction is the most common cause of intestinal obstruction and accounts for $\sim 80 \%$ of cases. ${ }^{4}$ By far the most common cause in the Western world is post-surgical adhesions, accounting for $\sim 75 \%$ of cases. In developing countries, incarcerated hernias account for the majority of cases. Other causes include gallstone ileus, foreign bodies, intussusception, intraluminal masses and malignancy (box 1).

The underlying cause of small bowel obstruction, particularly hernias, can be occasionally identified on plain films and should be looked for in cases of obstruction. It is imperative that the inguinal regions are covered on the abdominal radiograph, as these are the most common hernias to cause bowel obstruction. Obturator and umbilical hernias can sometimes be suspected from the gas pattern on plain films, with a low threshold for the prompt use of CT to elucidate further detail (figure 2). Gallstone ileus and abdominal masses causing obstruction may also occasionally be detected.

\section{lleus}

A paralytic or adynamic ileus refers to stasis of the bowel contents without a mechanical obstruction. Generally more loops of bowel are involved than present in a mechanical obstruction and a diffuse pattern of dilation is present, often involving the stomach, small bowel and colon. There are a number of causes, which include postoperative states, drugs, metabolic causes and intra-abdominal inflammation. It is often
Figure 1 Small bowel obstruction. (A) Multiple loops of gas-filled distended small bowel. Note the valvulae conniventes extending across the bowel circumferentially (arrow). (B) A paucity of abdominal gas due to fluid-filled obstructed small bowel. (C) An erect abdominal radiograph showing multiple fluid levels - 'step ladder' pattern (arrows).

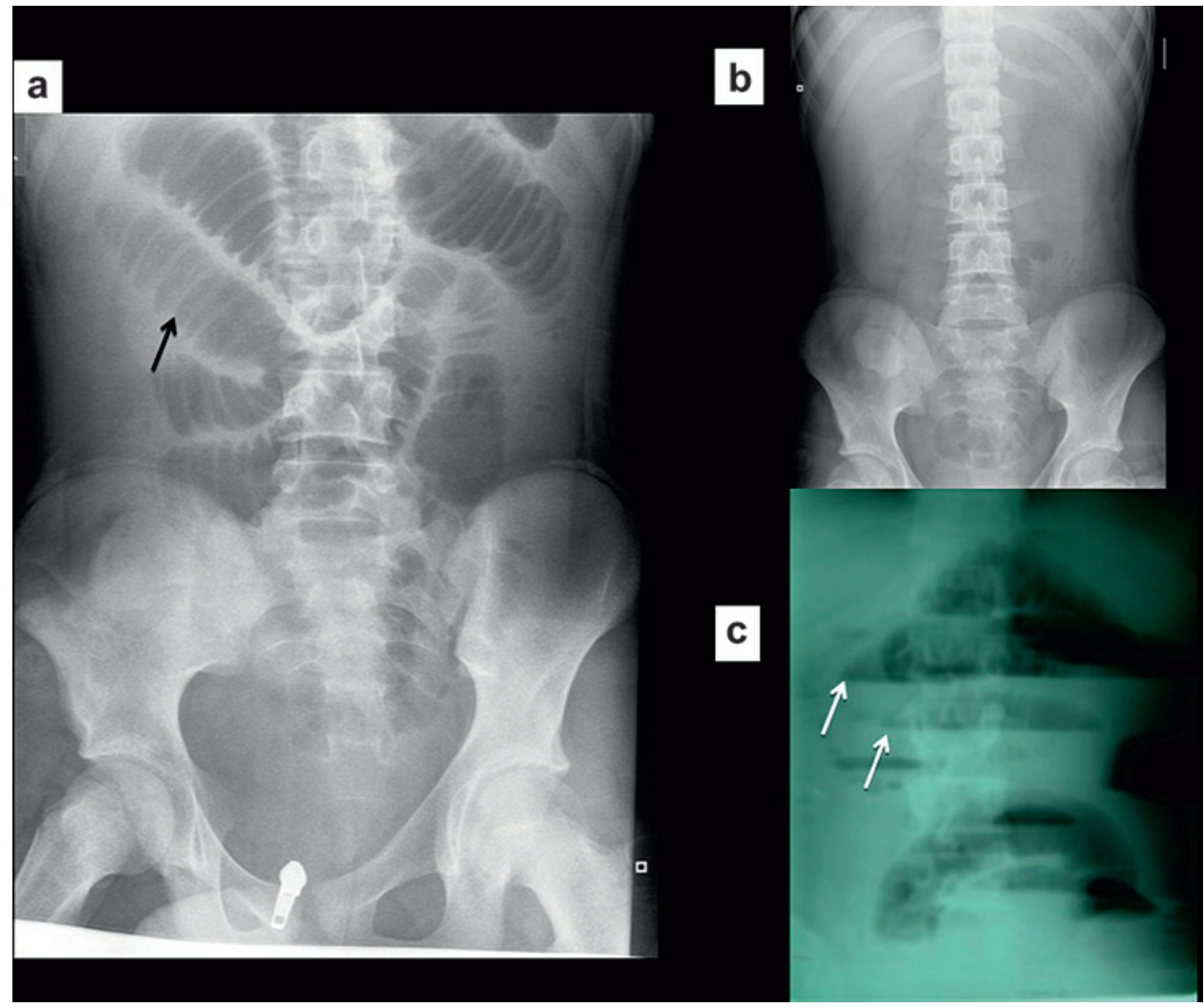




\section{Box 1 Common causes of small bowel obstruction}
1. Adhesions
2. Incarcerated hernias
3. Masses (including malignancy)
4. Volvulus
5. Intussusception
6. Gallstone ileus
7. Foreign body

difficult to distinguish between an ileus and mechanical bowel obstruction on a plain film and CT is often used in those in whom a mechanical cause is clinically suspected.

'Sentinel loop' is a term used to describe a localised ileus when one loop of bowel is distended. This commonly occurs adjacent to an area of intra-abdominal inflammation, such as in pancreatitis or appendicitis.

\section{Large bowel obstruction}

Large bowel obstruction accounts for the remaining 20\% of cases of intestinal obstruction. The most common cause is colorectal carcinoma. Other causes include metastatic deposits often from ovarian cancer, volvuli, diverticulitis, and rarely adhesions.

Conventional radiographs are often confirmatory in the diagnosis showing dilated loops of large bowel. The caecum distends to the greatest extent. When it reaches $80 \mathrm{~mm}$, there is a high risk of perforation (figure 3 ). An important consideration is whether there is associated small bowel dilation. If no gas is seen in the small bowel, this indicates that the ileocaecal valve is competent and increases the risk of associated perforation.

\section{Sigmoid volvulus}

This occurs when the sigmoid colon twists on its mesentery resulting in a closed loop obstruction. Patients may present with an acute abdomen or more insidious symptoms depending on whether the volvulus is causing partial or complete obstruction.
Ischaemia is a known complication. On plain radiograph, a massively dilated loop of sigmoid colon with no hautral markings is seen arising from the pelvis and extending up to the upper abdomen quadrant usually the right upper quadrant. This has been termed the 'coffee bean' sign (figure 4).

\section{Caecal volvulus}

Caecal volvulus is less common than sigmoid volvulus and accounts for less than $5 \%$ of large bowel obstructions. It usually occurs in middle-aged adults, typically in those who have not undergone previous surgery. Twisting typically occurs in the ascending colon just above the ileocaecal valve. The plain radiograph is usually diagnostic and shows a marked distended loop extending from the right iliac fossa into the left upper quadrant. The small bowel is most commonly distended and the distal large bowel is collapsed. A CT scan is rarely needed to confirm the diagnosis, but will reveal the twisted mesentery and may show an ischaemic segment or perforation (figure 5).

\section{Colitis}

The features of colitis on a plain radiograph include large bowel distension, wall thickening as a result of mucosal oedema, and irregularity of the bowel wall termed 'thumb-printing'. Toxic megacolon is the hallmark of severe colitis, which is characterised by marked colonic dilation (figure 6). A colonic diameter of $>55 \mathrm{~mm}$ is at risk of perforation. Acute ulcerative colitis is by far the most common cause. Others include infective, pseudomembranous and ischaemic colitis.

\section{Gastric outlet obstruction}

A massively distended stomach is the characteristic finding in gastric outlet obstruction and can be readily detected on plain films (figure 7). Gastric outlet obstruction is most commonly caused by gastric ulceration. Other causes include malignancy (involving either the stomach or pancreas), polyps, congenital lesions, volvulus or pancreatic pseudocysts. A dilated stomach may also occur in diabetic neuropathy, when no structural abnormality is present.
Figure 2 Small bowel obstruction due to obstructed obturator hernia. (A) Distended loops of small bowel. Also note a small amount of gas projecting beyond the inguinal ligament within the hernial orifice on the right (short black arrow). (B,C) Coronal and axial CT images show bowel loop between the obturator internus and externus muscles confirming an obstructed right obturator hernia (long black arrow).

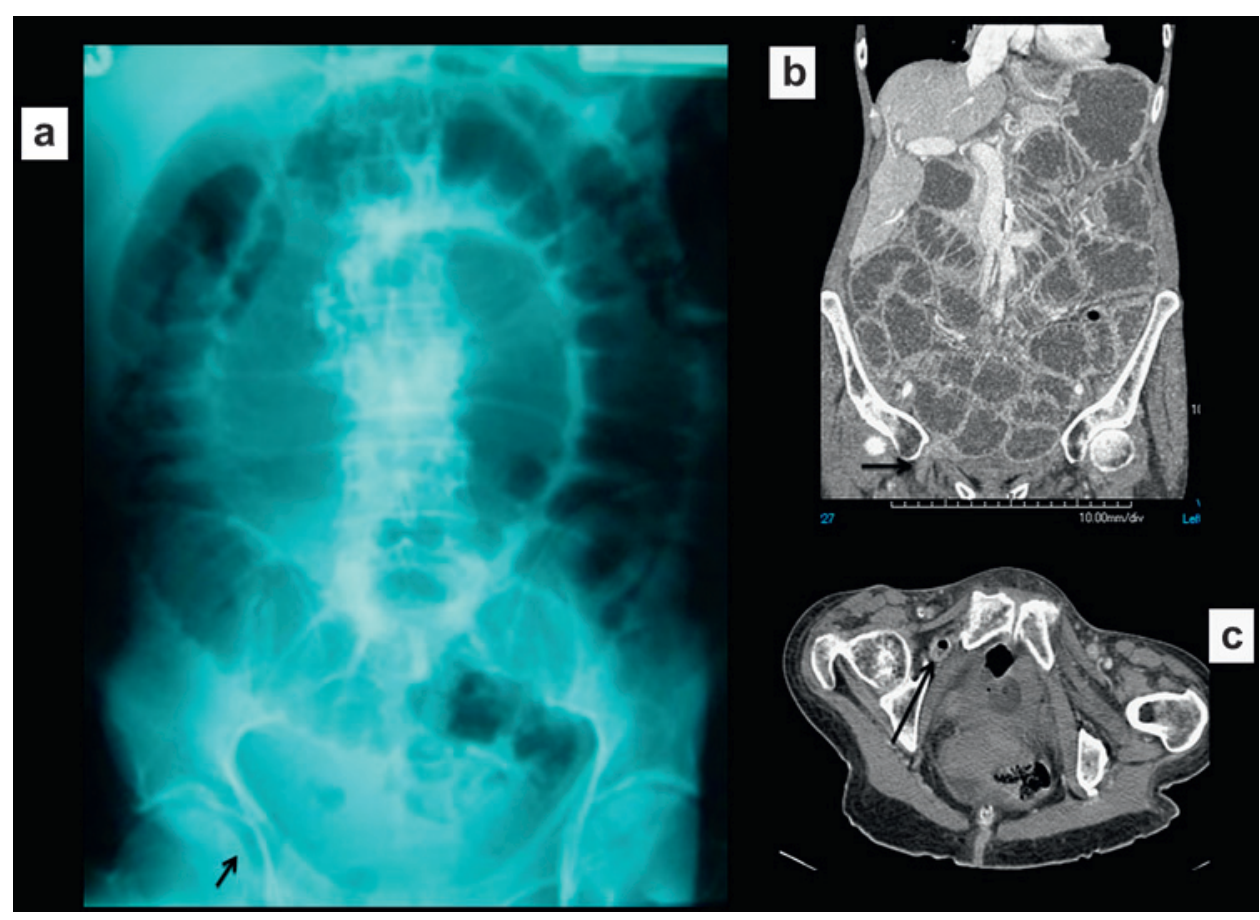


Figure 3 Large bowel obstruction. large bowel loops. Note grossly dilated caecum (white arrow). (B) Subsequent CT scan showing dilated large bowel with a transition point in the pelvis due to an obstructing carcinoma of the sigmoid colon (white arrow). (A) Plain radiograph showing distended

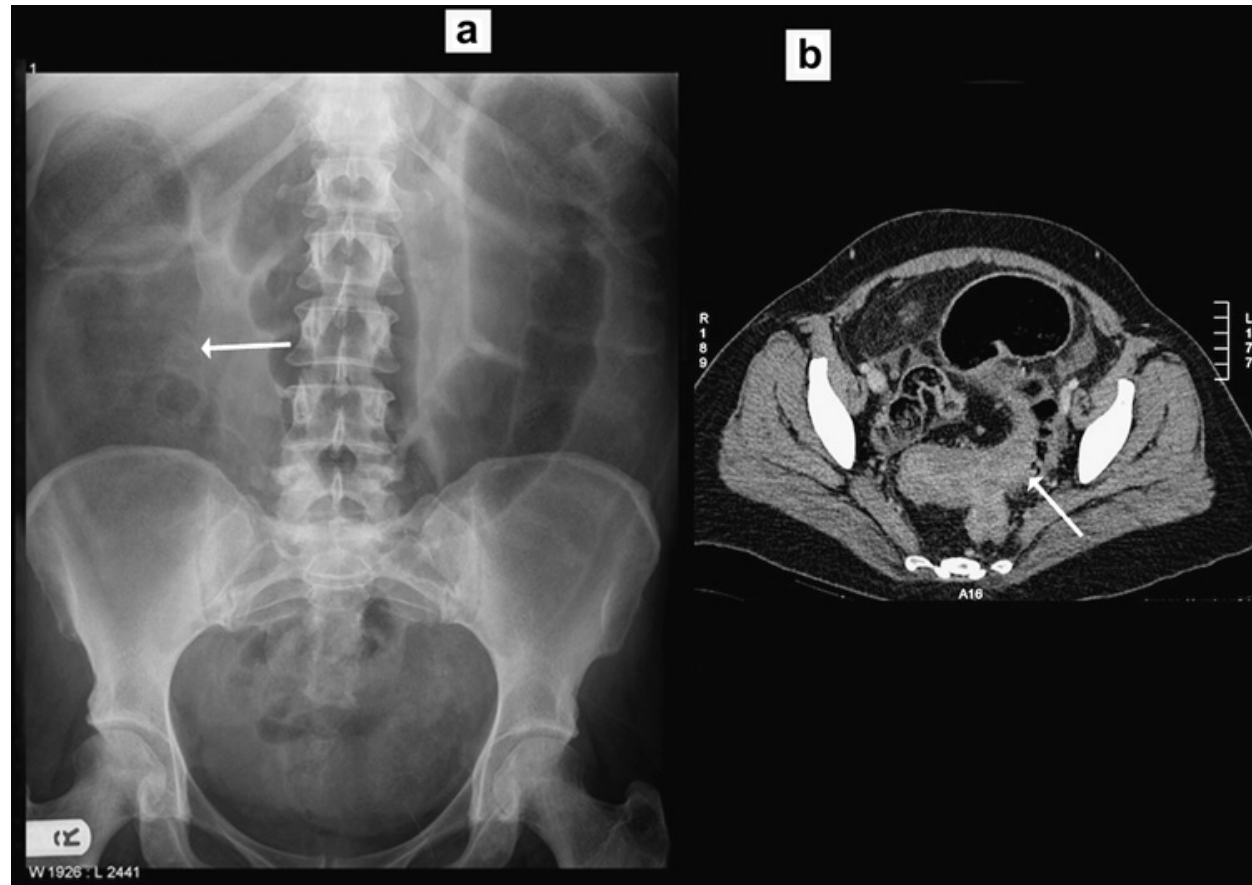

\section{Gas within other luminal structures - the biliary tree and portal vein}

Gas in the biliary tree (pneumobilia) is represented as a central linear branching pattern over the liver. It can be distinguished from gas in the portal vein, as this extends out to the periphery of the liver. CTwill differentiate portal venous gas from biliary gas if there is diagnostic doubt. There are a number of causes of gas both in the biliary tree and the portal veins outlined below (table 2).

\section{Previous biliary intervention}

The most common cause of air in the biliary tree is an incompetent sphincter of Oddi, often seen after sphincterotomy during endoscopic retrograde cholangiopancreatography (ERCP) and is of no clinical consequence. Other forms of biliary intervention and surgery will produce the same picture.

\section{Gallstone ileus}

Gallstone ileus results in air in the biliary tree. This occurs when a large gallstone erodes though the gallbladder wall and into the small bowel. It most commonly lodges in the distal ileum, resulting in small bowel obstruction. The classic triad of radiological features on plain film are dilated small bowel, gas in the biliary tree (pneumobilia) and a calcified gallstone.

These features are only seen in about $50 \%$ of cases (figure 8 ). Gallstone ileus is an important cause of small bowel obstruction, which should be considered particularly in older female patients.
Figure 4 Sigmoid volvulus. (A) Plain film showing a large distended loop of sigmoid colon arising from the pelvis extending up to the right upper quadrant, with proximal large bowel obstruction. This is the typical appearance of sigmoid volvulus (coffee bean sign; arrows). (B,C) A confirmed sigmoid volvulus on the CT scan. Note the 'bird's beak' appearance (white arrows) of the twisted sigmoid colon mesentery.

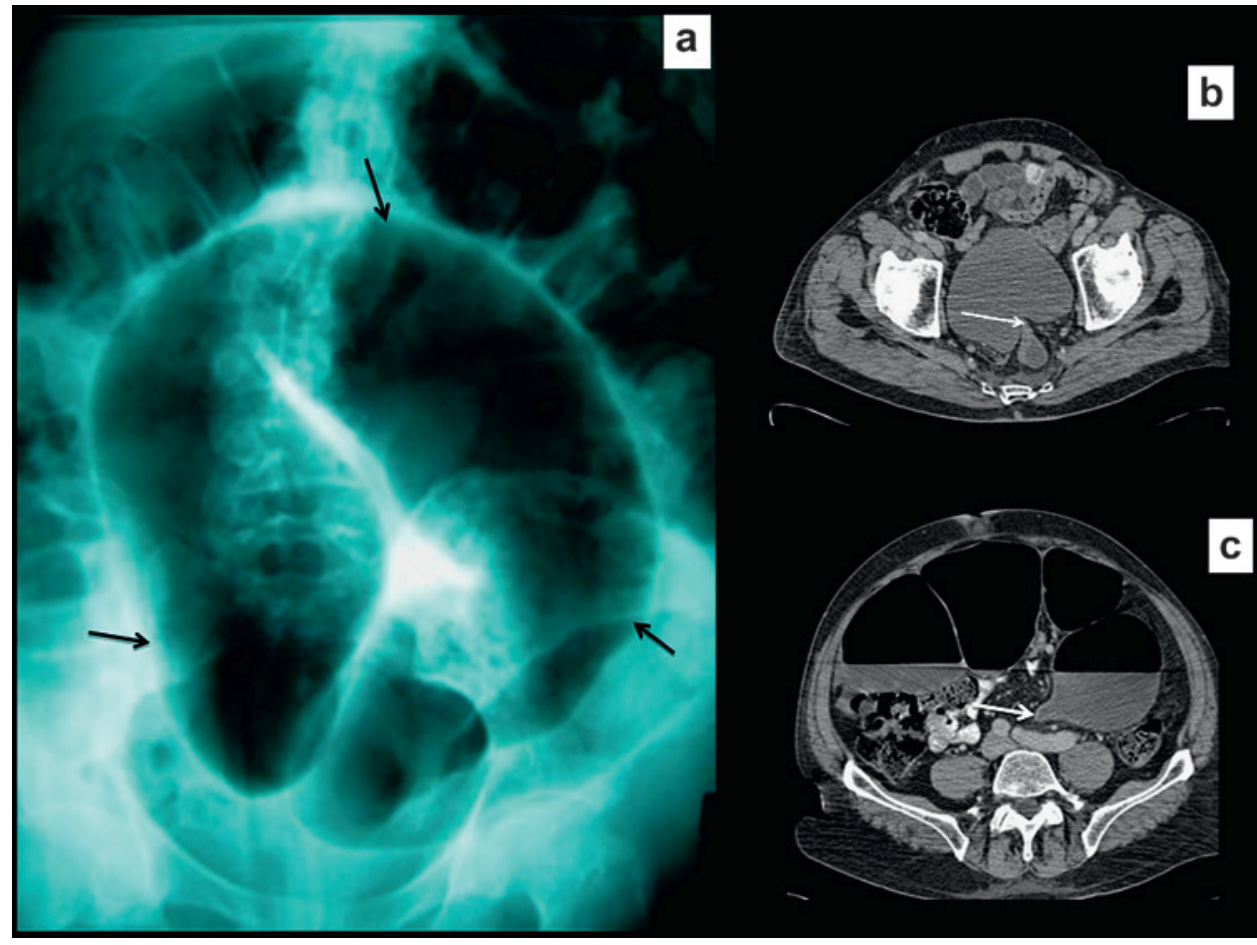


Figure 5 Caecal volvulus. (A) Erect abdominal radiograph showing a grossly dilated gas-filled loop of bowel (caecum) projected in the upper abdomen (white arrow). Note the lack of gas in the rest of the large bowel and dilated small bowel loops (black arrow). $(B, C)$ This was confirmed on the CT scan, which also shows twisted caecal mesentery.

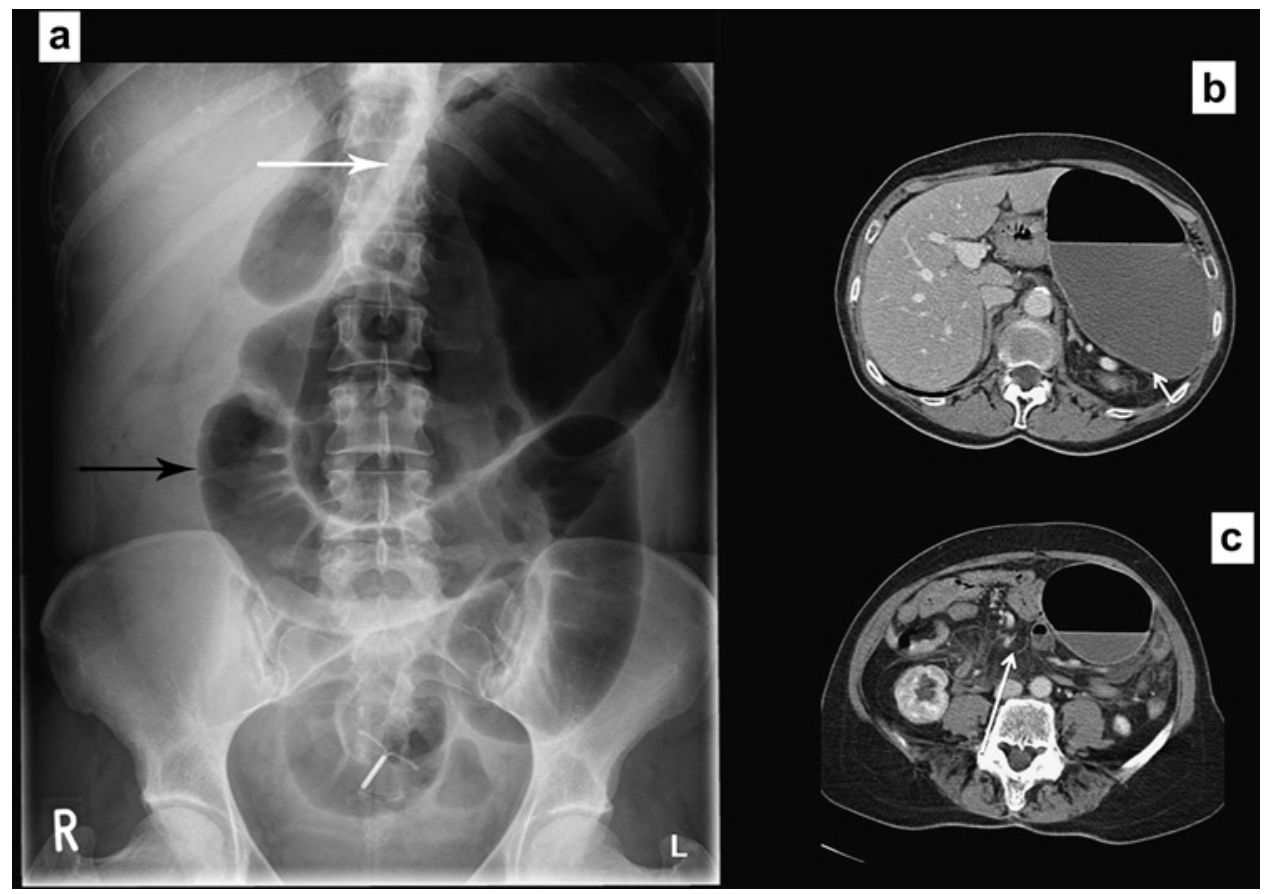

\section{Necrotising enterocolitis}

In neonates, portal vein gas is commonly associated with necrotising enterocolitis. This is an inflammatory condition of the small and large bowel, which predominantly affects premature neonates usually within the first weeks of life. It is a gastroenterological emergency and may result in bleeding, erosion and perforation. Evidence of gas within the bowel wall, depicted as a bubbly appearance of bowel loops, supports this diagnosis. CT in this age group is generally avoided in view of the high radiation dose.
Ischaemic bowel

In adults, portal vein gas is most commonly associated with ischaemic bowel, which has a high mortality exceeding 50\% (figure 9). Other plain film findings include gas in the bowel wall as described below.

\section{Gas in hollow viscera}

Gallbladder empyema

Air in the gallbladder can result from infection. While not specifically a test used in the investigation of biliary disease, the
Figure 6 Colitis. (A) Plain radiograph showing dilated loop of transverse colon (white arrow), consistent with toxic megacolon, and featureless oedematous descending colon secondary to inflammatory bowel disease. (B) Plain radiograph of a different patient showing a long segment of thick walled and oedematous descending colon (white arrows). (C) This was confirmed on the CT scan (white arrow).

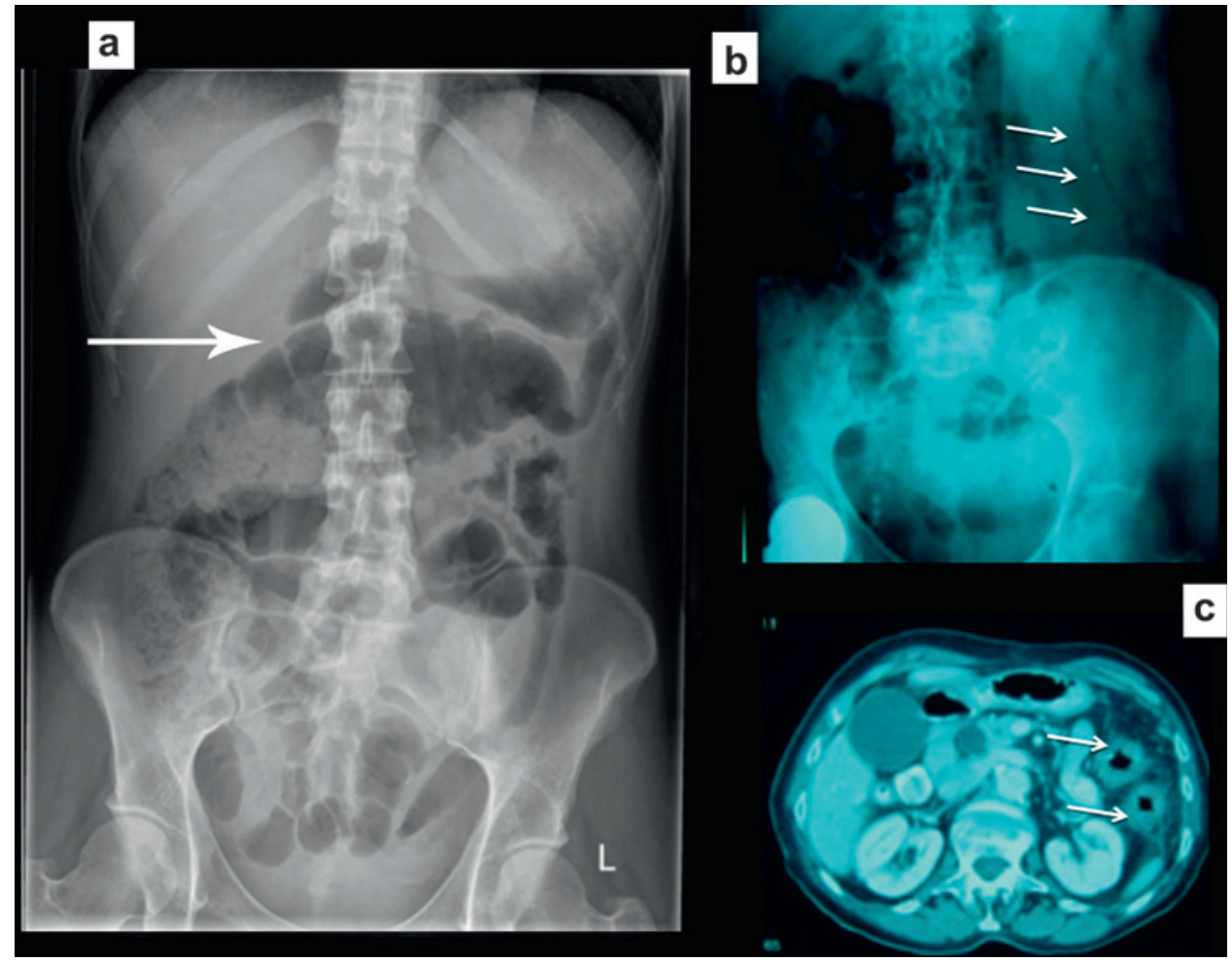


Figure 7 Grossly dilated stomach. (A) Plain film showing a distended gas-filled stomach (black arrow). (B,C) Coronal and sagittal CT scans confirming that this is due to gastric outlet obstruction. Note the nasogastric tube in the stomach (white arrow).

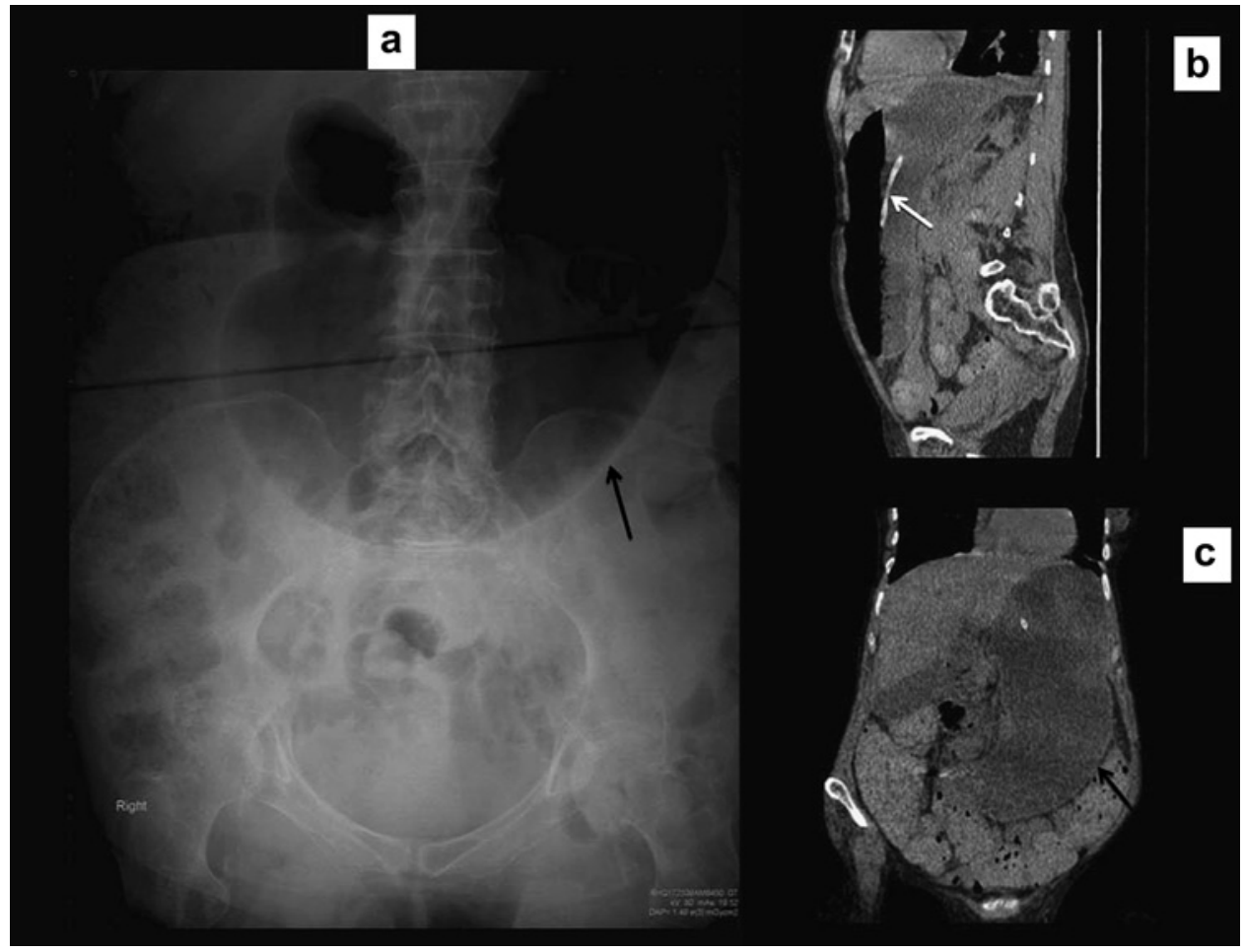

plain film may show an air fluid level that is suggestive of empyema of the gallbladder. Further imaging with ultrasound or CT is usually performed (figure 10). Empyema of the gallbladder is a surgical emergency and requires urgent drainage, which is often performed via the transhepatic approach under ultrasound guidance. Other causes of air in the gallbladder include gallstone ileus and emphysematous cholecystitis, which may also cause intramural gas.

\section{Urinary tract gas}

Gas in the urinary bladder is most commonly seen as a result of catheterisation. It can also be seen in cases of gas-forming infection or a vesicoenteric fistula, typically in association with diverticular disease. Gas can occasionally be seen extending into the ureter from the urinary bladder.

\section{Bowel gas displacement}

\section{Abdominal masses}

Although there is a broad variation of bowel gas distribution, not least due to variable fluid content in the small bowel, the displacement or atypical distribution of bowel gas may suggest soft tissue pathology in the abdomen. Bowel gas displacement is identified in the presence of organomegaly or the presence of a pathological mass within the abdomen, such as a large ovarian cyst or renal tumour (figures 11 and 12). Although plain film is not indicated to specifically look for bowel gas displacement in suspected organomegaly or malignancy, these findings may be detected when investigating other symptomatology.

\section{Ascites}

The presence of a large volume of ascites causes the displacement of bowel as it congregates or 'floats' in the central abdomen. This produces a typical pattern on plain film. This is often investigated further with ultrasound or CT, which will often determine the cause, which is often liver disease or malignancy (figure 13).

\section{Exraluminal gas patterns}

Intraperitoneal perforation

The most commonly occurring and simplest form of extraluminal gas to identify is a pneumoperitoneum. This encompasses any gas within the peritoneal cavity, typically due to a perforated gas-filled viscus. Sigmoid and duodenal perforations are the usual culprits, but other important considerations include recent surgery, post procedural, such as laparoscopy, or after controlled instrumentation, such as the use of a continuous ambulatory peritoneal dialysis catheter. Pneumoperitoneum is more easily recognised on an erect chest radiograph, hence the commonality of requesting these two radiographs in conjunction. Nevertheless, there are several well-described signs on the supine abdominal radiograph. These include air under the diaphragm, Rigler's sign, the falciform ligament sign, triangles of gas, and the football and continuous diaphragm signs.

Air under the diaphragm is strictly speaking a chest radiograph diagnosis, with the falciform ligament and Rigler's signs a perforation. Rigler's is also known as the 'double wall sign', as the bowel wall is crisply visualised because of gas being present on either side (within the bowel as normal) and outside the bowel (in the peritoneum). The falciform ligament sign similarly occurs, as gas on either side of it outlines an otherwise unidentifiable plain radiograph structure. Triangles of gas occur, as free peritoneal air is trapped between opposed bowel loops

Table 2 Causes of portal vein and biliary tree gas

\begin{tabular}{lll}
\hline \multicolumn{2}{l}{ Causes of portal vein gas } & \\
\cline { 1 - 2 } Children & Adults & Causes of biliary tree gas \\
\hline NEC & Ischaemic bowel & ERCP \\
Umbilical vein catheters & Necrotic colorectal cancer & Biliary stents and surgery \\
Postoperative states & Post-endoscopy/barium & Gallstone ileus \\
& enemas & Trauma \\
& Intra-abdominal sepsis, & Duodenal ulcer perforating \\
& ie, diverticulitis & the common bile duct \\
\hline
\end{tabular}

ERCP, endoscopic retrograde cholangiopancreatography; NEC, necrotising enterocolitis. being by far the most common abdominal radiographic signs of 
Figure 8 Gallstone ileus. (A) Plain radiograph showing the central distribution of gas in the biliary tree (short black arrow). (B) Plain radiograph showing small bowel obstruction to the level of a gallstone (long black arrow). $(C, D) C T$ scans showing air within the biliary tree (long white arrow) and an obstructing gallstone in the distal ileum (short white arrow).

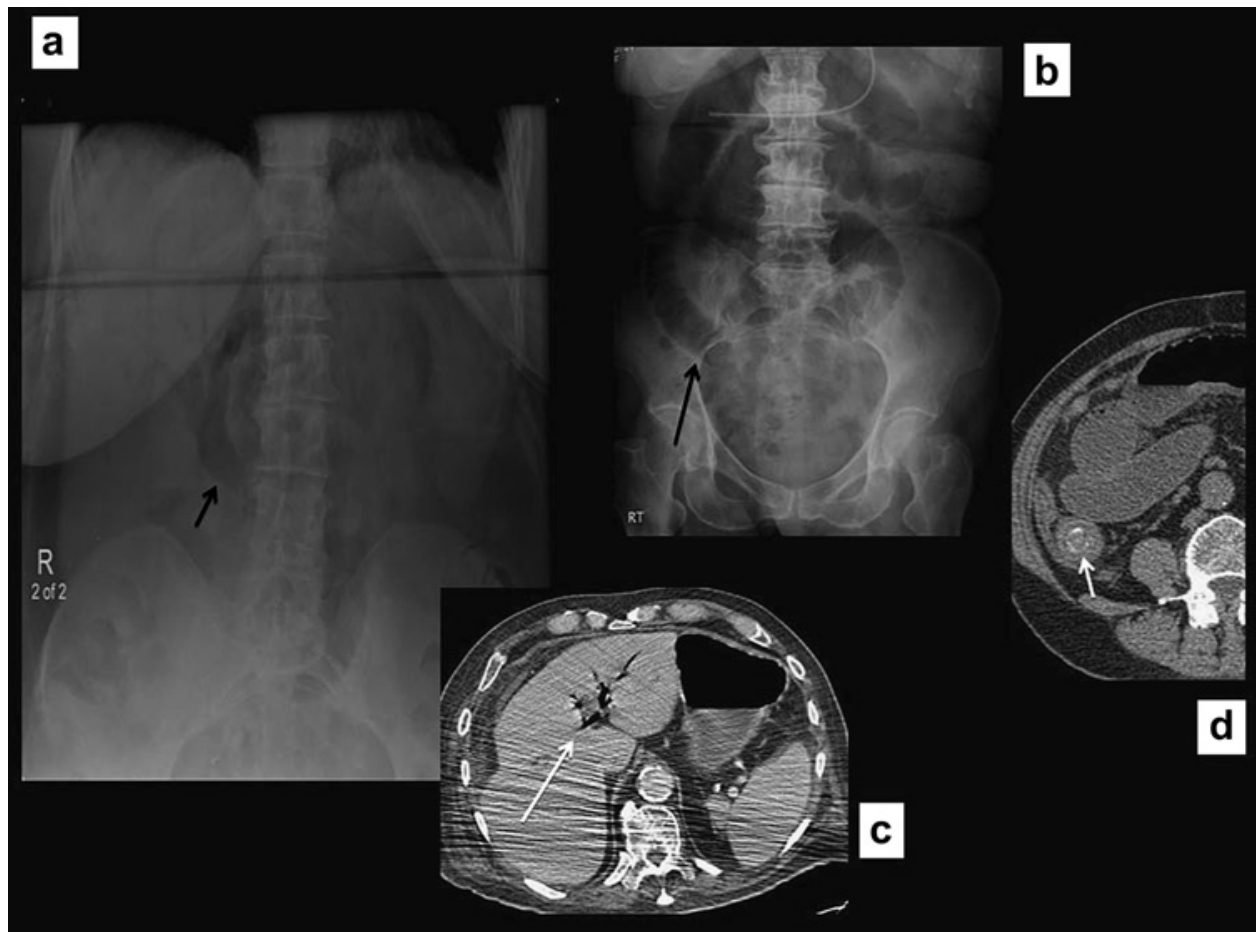

(figures 14 and 15). The rarely identified football sign gains its name from the football shaped appearance of gas outlining the peritoneum in the upper abdomen.

\section{Retroperitoneal perforation}

Much less commonly, free gas is present within the retroperitoneum. This occurs with selected perforations of the duodenum and sigmoid colon, in particular due to iatrogenic injury during ERCP. This is best identified as gas outlining either the kidney or psoas muscle (figure 16).

\section{Intramural gas}

Pneumotosis coli and ischaemic bowel

Rarely, gas enters the wall of a viscus, such as the colon, producing an intramural gas pattern on plain films. This may be incidental and non-life threatening, such as in pneumatosis coli, or indicative of serious life-threatening pathology, as with ischaemic colitis or toxic megacolon with impending perforation. Intramural gas typically has a 'bleb-like' appearance due to tiny pockets of gas within the wall. This is far more sensitively detected with CT (figure 17). CT may show other associated
Figure 9 Portal gas. $(A, B)$ Extensive portal gas in an adult patient after traumatic nasogastric tube insertion (short black arrow) and splenic vein (long black arrow). This is confirmed on a CT scan (long white arrow). Note the peripheral distribution (gas extends all the way up to the periphery of liver) of portal gas. (C) Plain radiograph of a neonate in a special baby care unit showing gas within the portal vessels (short white arrow) secondary to necrotising enterocolitis.

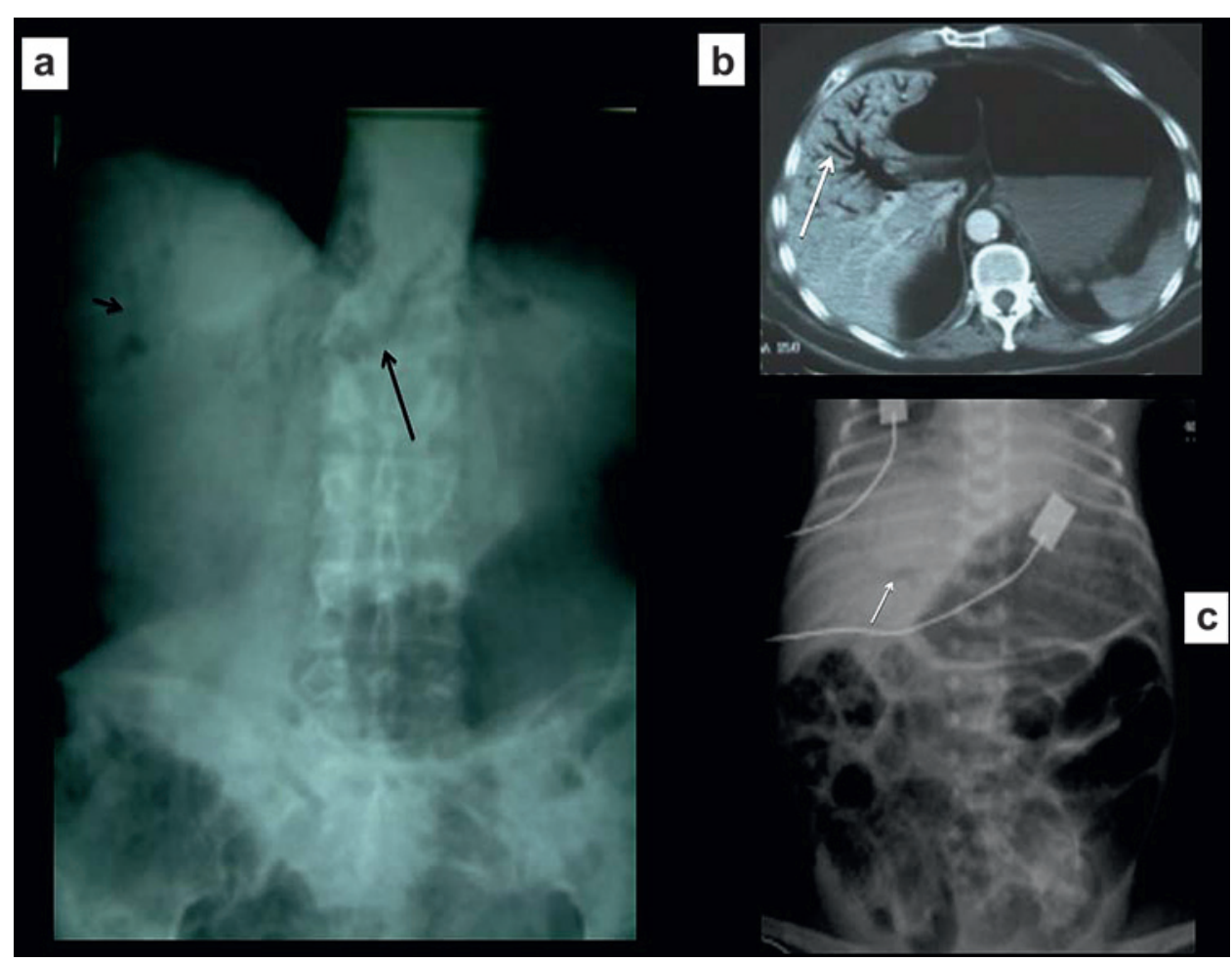


Figure 10 Gas in the gallbladder. (A) Air fluid level within the gallbladder (black arrow) consistent with a gallbladder empyema. (B) Intramural gas in emphysematous cholecystitis on plain radiograph. Note the difference between gallbladder empyema and emphysematous cholecystitis.

(C) Ultrasound confirms gas in the wall of the gallbladder (arrow).

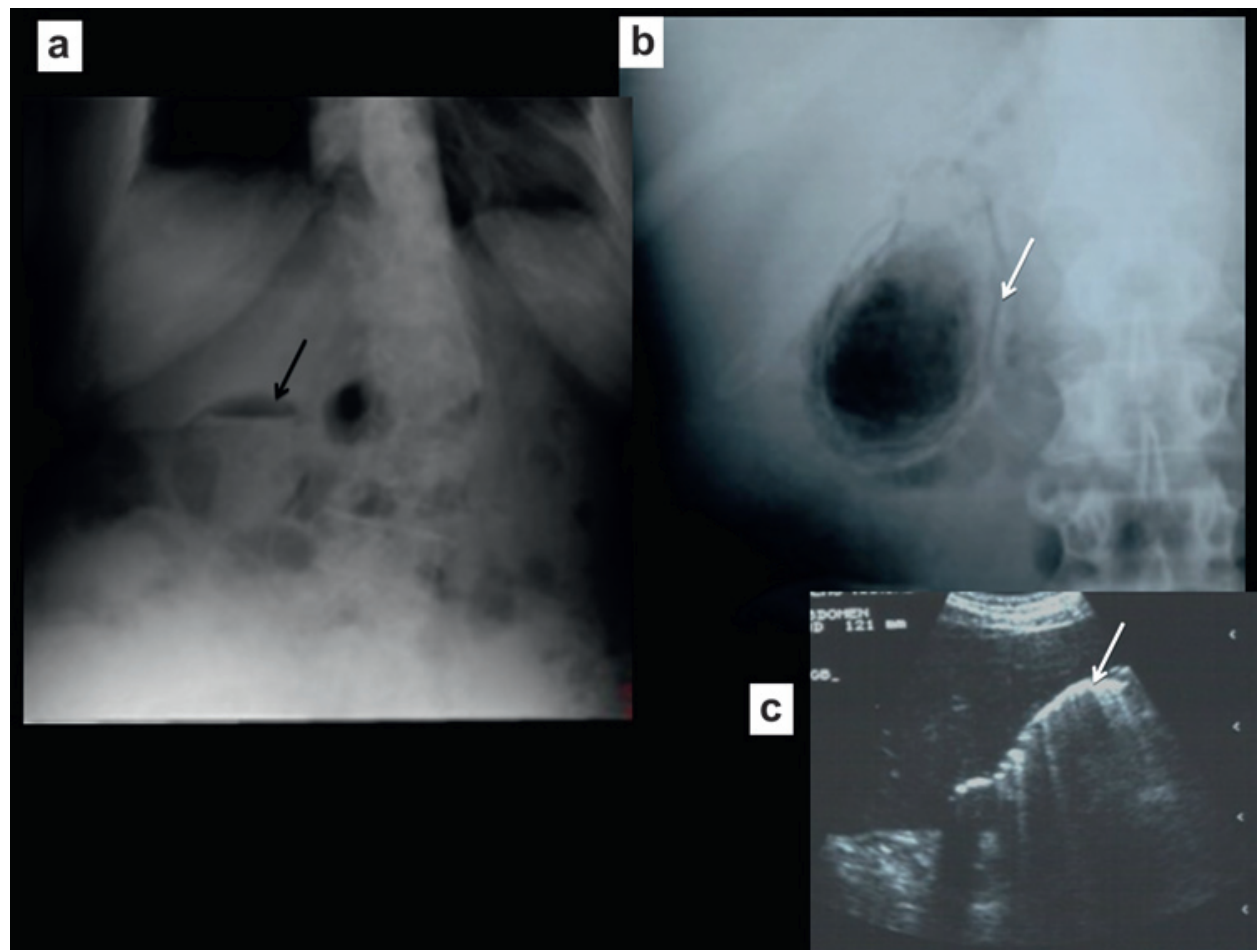

findings, such as bowel wall thickening or dilation, and lack of contrast enhancement in the affected segment and may also depict thrombus within the mesenteric vessels.

\section{Gas-forming infection}

Intramural gas may be present within the wall of other intraabdominal structures, such as the urinary bladder, gallbladder or aorta. Infection due to gas-forming organisms, particularly in diabetics, is the usual cause. ${ }^{4}$ Figure 10 shows air in the gallbladder wall in addition to air within the gallbladder itself. Figure 18 shows air within the kidney in a case of emphysematous pyelonephritis.

Superficial collections, abscesses and wound infections containing gas may similarly be evident on plain radiograph, usually depicted by a pattern of multiple different shaped gas locules. The clinical consequences of this can be serious, and prompt recognition is important (figure 19).

On occasion, usually in postoperative patients, intra-abdominal collections, such as subphrenic abscesses, can be visualised on plain radiograph. This may be either due to gas contents within the collection or displacement of normal bowel gas due to mass effect, or both (figure 20).

\section{Common misdiagnoses and conundrums}

A common misperception is that air in the rectum means that there is no bowel obstruction - the reasoning behind this being if the bowel is obstructed no air can pass through. This theory is, however, flawed, as obstruction of the bowel does not mean the
Figure 11 Bowel gas displacement $\mathrm{I}$. (A) Large abdominal aortic aneurysm (arrows) displacing the left-sided colonic bowel gas. (B) Massive splenomegaly (arrows) displacing the bowel gas to the right and inferior aspects of the abdomen.

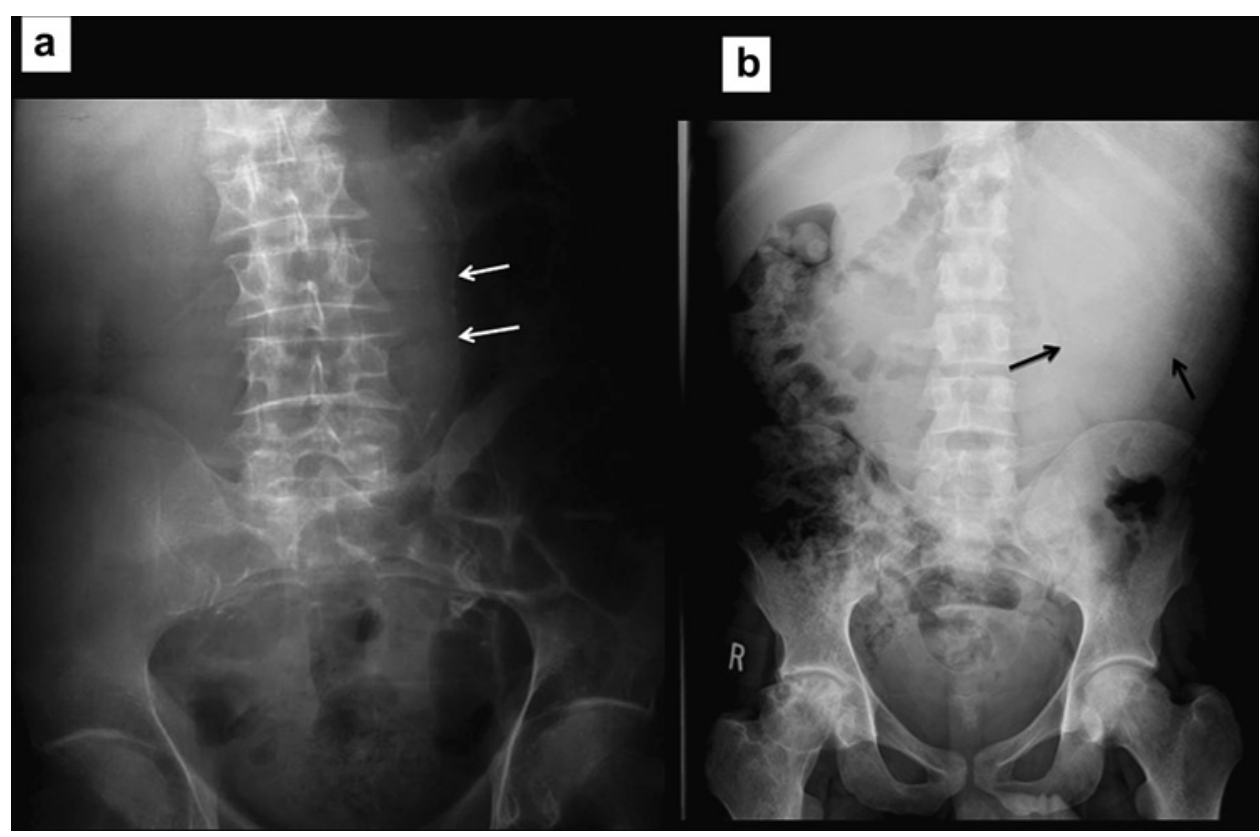


Figure 12 Bowel gas displacement II. (A) A mass (arrows) arising from the pelvis displaces bowel gas superiorly. (B) CT confirms this to be a large ovarian mass. (C,D) Massive hepatosplenomegaly (arrows) pushing the bowel gas inferiorly. This is confirmed on CT.

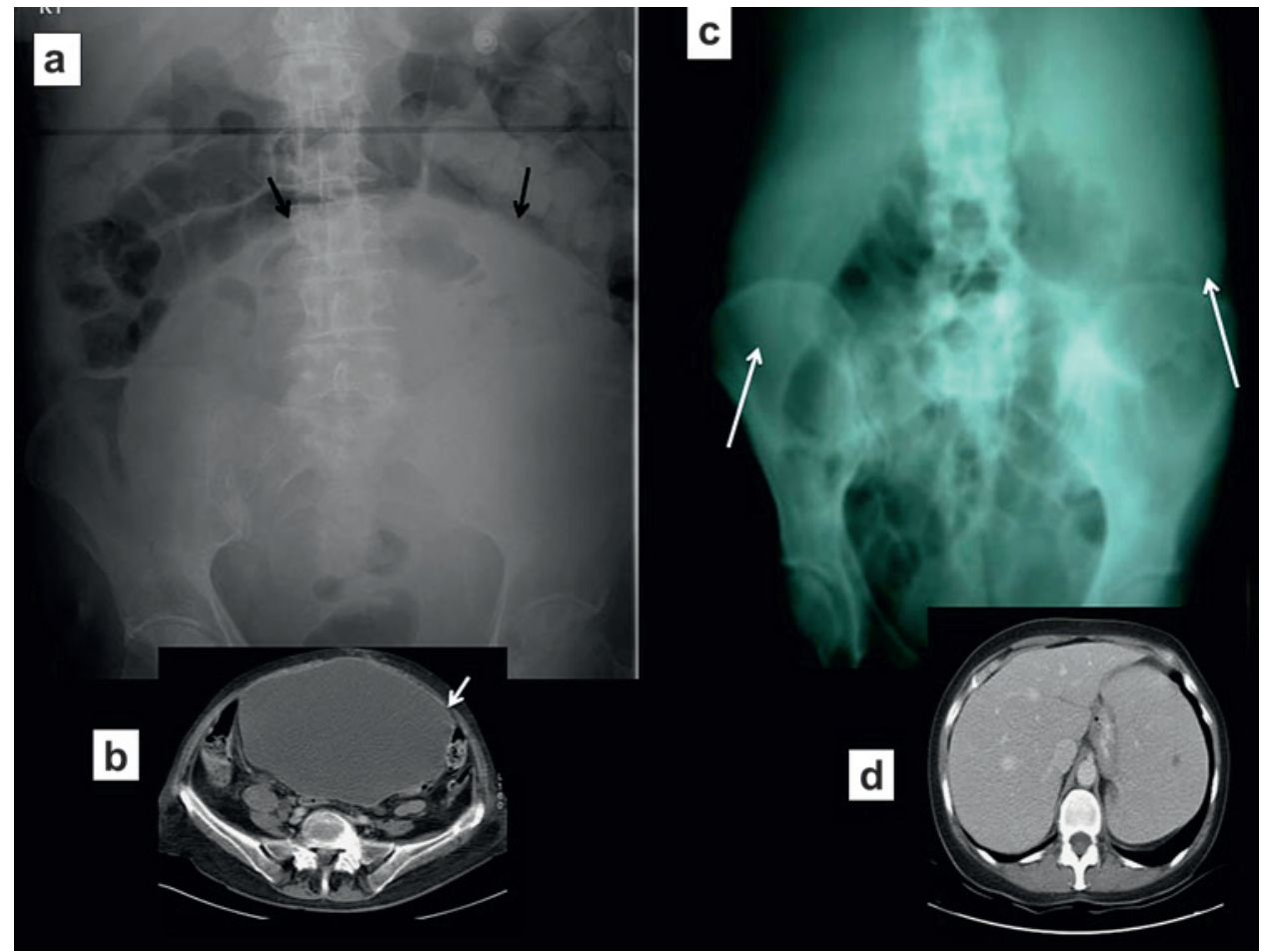

immediate disappearance of distal gas. A partial obstruction will allow the passage of air distally, and, in complete obstruction, fermentation of the residual faecal matter in the large bowel can produce gas distal to the obstruction for some time after the bowel becomes completely obstructed. Air may also be introduced during digital rectal examination, before the radiograph being performed. For these reasons, gas in the rectum does not exclude bowel obstruction, and a collapsed large bowel is generally a more reliable sign.

It can often be difficult to determine an ileus from a mechanical obstruction on a plain film. If there are also associated fluid-filled loops, these are not clearly depicted and this can make interpretation difficult. In addition, it can be difficult to determine the exact site of obstruction, particularly in the large bowel, as the loop just proximal to the obstruction can be filled with fluid and or faeces.

Perhaps the most common plain film conundrum in managing the acute abdomen is that of free intraperitoneal air under the diaphragm on chest x-ray. It can be mimicked by atelectasis at the lung bases or occasionally Chilaiditi syndrome. Chilaiditi syndrome is the result of colon or, less commonly, small bowel becoming interposed in the hepatodiaphragmatic space. The most common reasons for this are laxity of the hepatic and intestinal suspensory ligaments, redundant colon, and paralysis
Figure 13 Bowel gas displacement III. (A) Plain radiograph showing centrally placed bowel loops (arrows) of normal calibre. (B) CT confirms the presence of gross ascites.

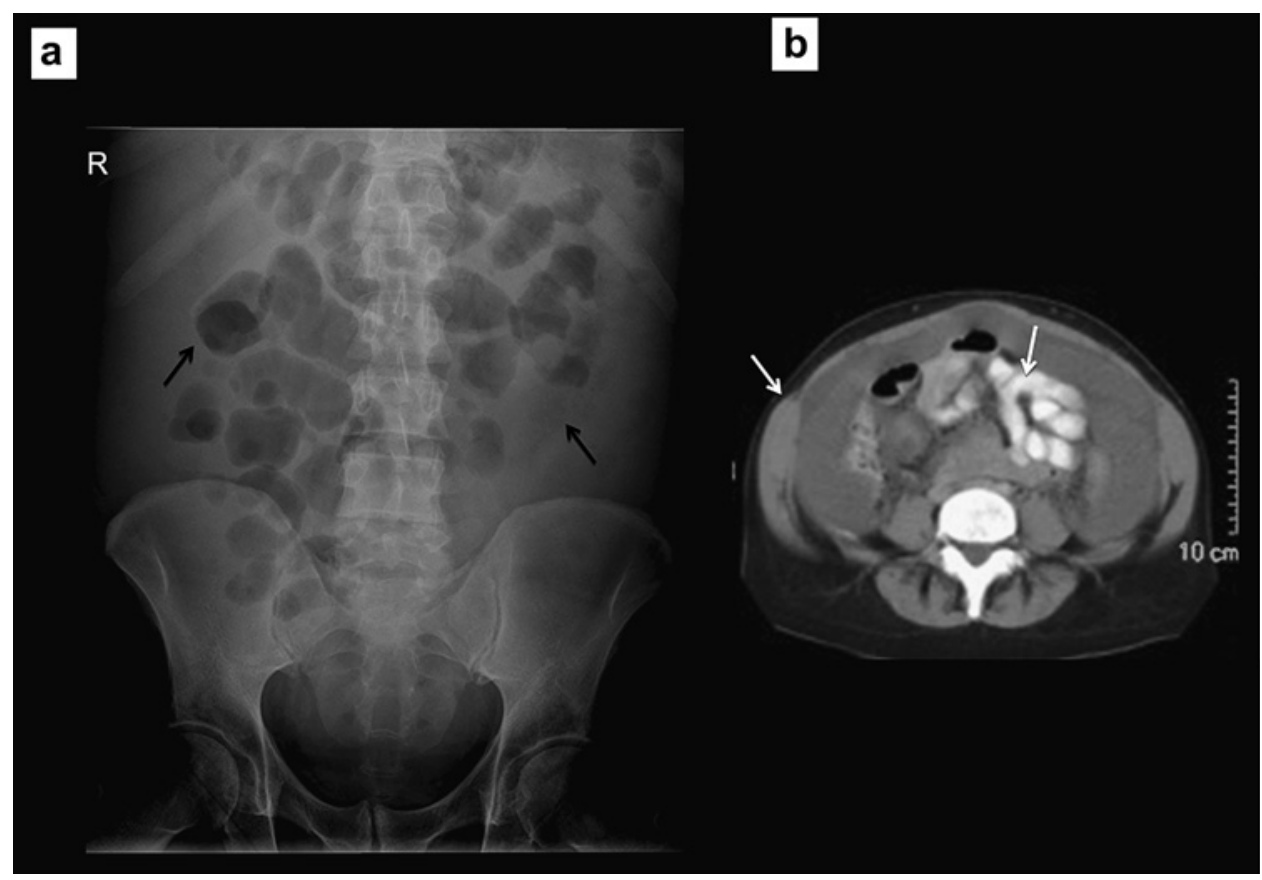


Figure 14 Signs of pneumoperitoneum I. (A) Free subdiaphragmatic gas on chest radiograph (arrows). (B) Triangle of free peritoneal gas (arrows). (C,D) Abdominal radiograph and complementary CT scan showing the falciform ligament sign (arrows).

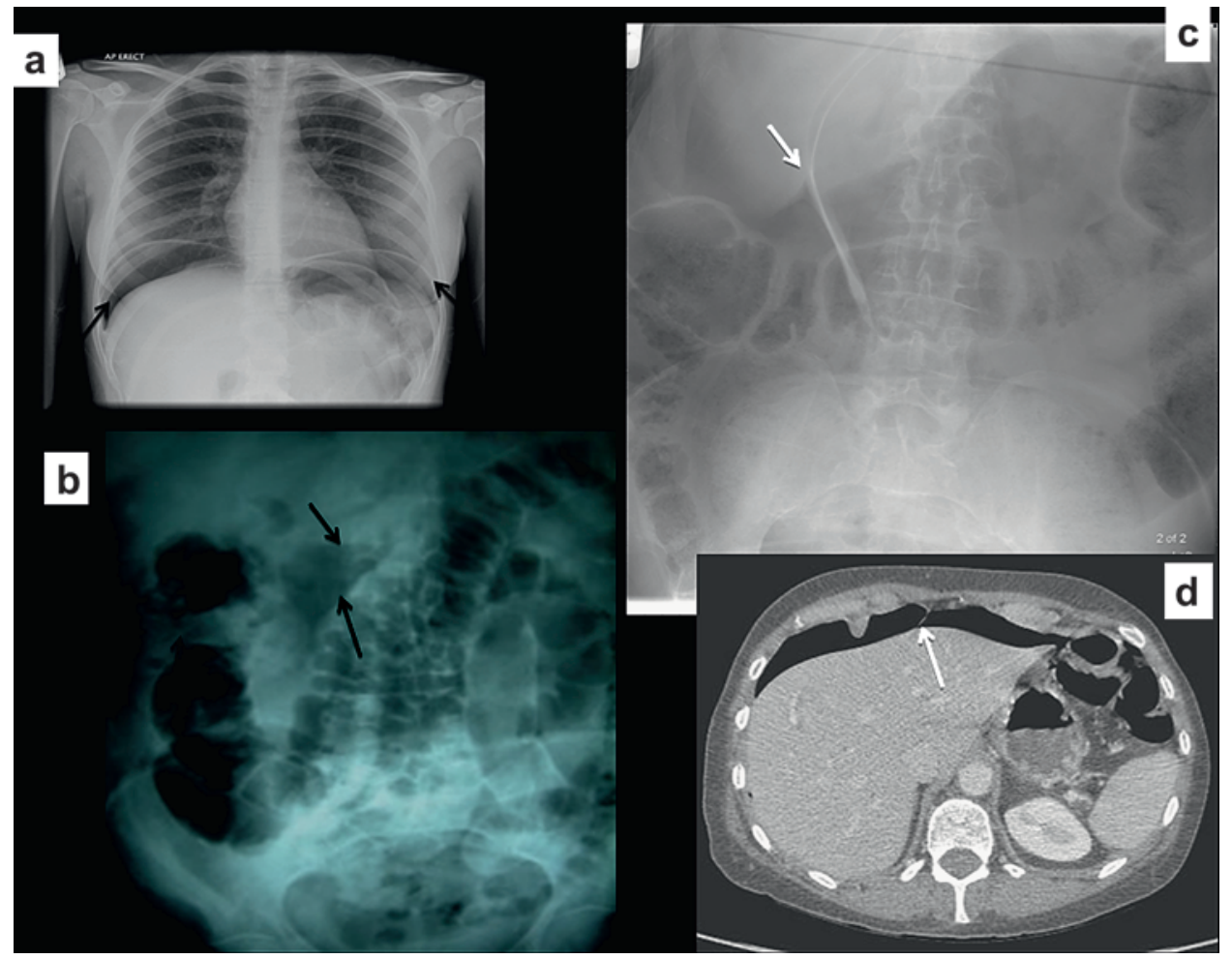

Figure 15 Signs of

pneumoperitoneum II. (A) Rigler's sign (arrow) showing air on both sides of the bowel wall. (B) Left lateral decubitus radiograph showing small amount of free gas (arrow) confirms bowel perforation. significantly higher than that of a standard chest x-ray (typical dose $0.02 \mathrm{mSv}$ ), and this should be taken into account when requesting serial examinations, particularly in young patients. The radiation dose of abdominal CT is $10-100$ times greater than that of an abdominal film at $\sim 10 \mathrm{mSv}$. As described in this pictorial review, abdominal radiographs may reveal abnormalities highly suggestive of specific diagnoses. They do, however have a low diagnostic accuracy in the evaluation of abdominal pain. For example, plain radiographs only detect $\sim 50 \%$ of cases of small bowel obstruction. ${ }^{56}$ CT is much more sensitive and reveals the cause of small bowel obstruction in $70-90 \%$ gency setting, as it is quick and technically easy to perform. The radiation dose is $\sim 0.1-1.0 \mathrm{mSv}$ for an abdominal film. This is

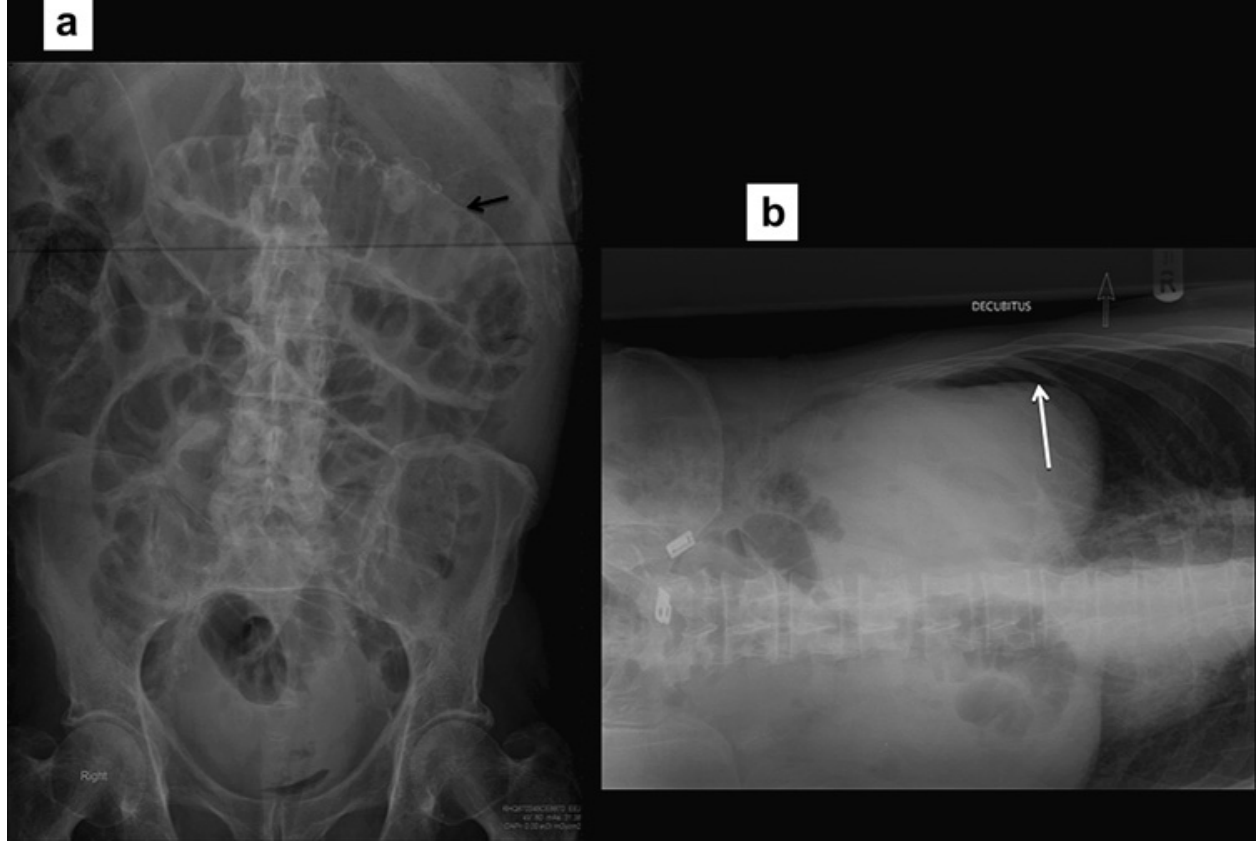


Figure 16 Retroperitoneal free gas. (A) Abdominal radiograph showing air outlining the left psoas muscle (arrows). (B) CT scan showing free gas (arrow) in the pelvis after rigid sigmoidoscopy causing sigmoid perforation. (C) Gas from the pelvis tracking through the retroperitoneum to lie around the left kidney (arrow).

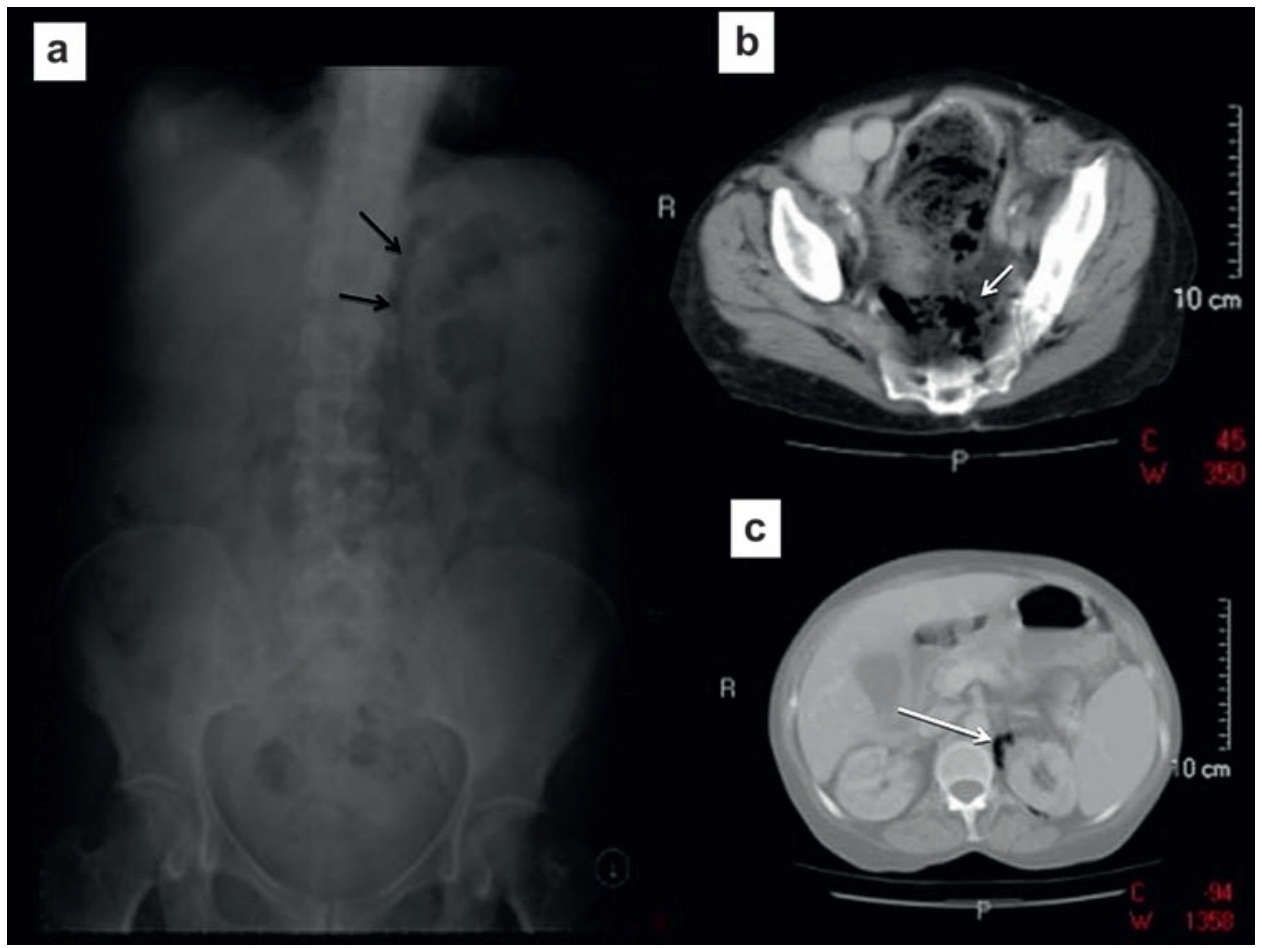

of cases. ${ }^{5}{ }^{6}$ More recent data suggest this sensitivity is even higher and well above $90 \%$, probably as a result of the use of multidetector $\mathrm{CT}^{7-9}$

CT is also much more sensitive in the diagnosis of pneumoperitoneum than conventional radiographs. Erect chest x-rays are better than abdominal films, but are insensitive to small locules of gas measuring $1 \mathrm{~mm}$ and only around $33 \%$ sensitive to locules measuring 1-13 mm. ${ }^{10}{ }^{11}$ Another major advantage is that the site can often be identified or inferred. Clues include a concentration of extraluminal gas bubbles, thickening or inflammatory change, contrast leakage and, on occasions, a segmental defect in the bowel wall from the location of the locules of air, associated inflammatory change or contrast leakage. Generally speaking, air seen around the liver suggests an upper gastrointestinal perforation, while air seen in the pelvis, inframesocolic or supramesocolic regions suggests perforation of the colon or appendix. Accuracy of multidetector CT in determining the site of perforation is quoted to over $80 \%{ }^{9} 1112$

Plain films have also been shown to be of little value in detecting other common causes of abdominal pain such as
Figure 17 Intramural gas: bowel. (A) Streaky intramural gas within the ascending colon on plain radiograph (see the magnified image); laparotomy confirmed ischaemic colitis. (B) Bleblike intramural air on a routine barium enema study and (C) on CT in the ascending colon. This was an incidental finding and likely to represent pneumatosis coli.

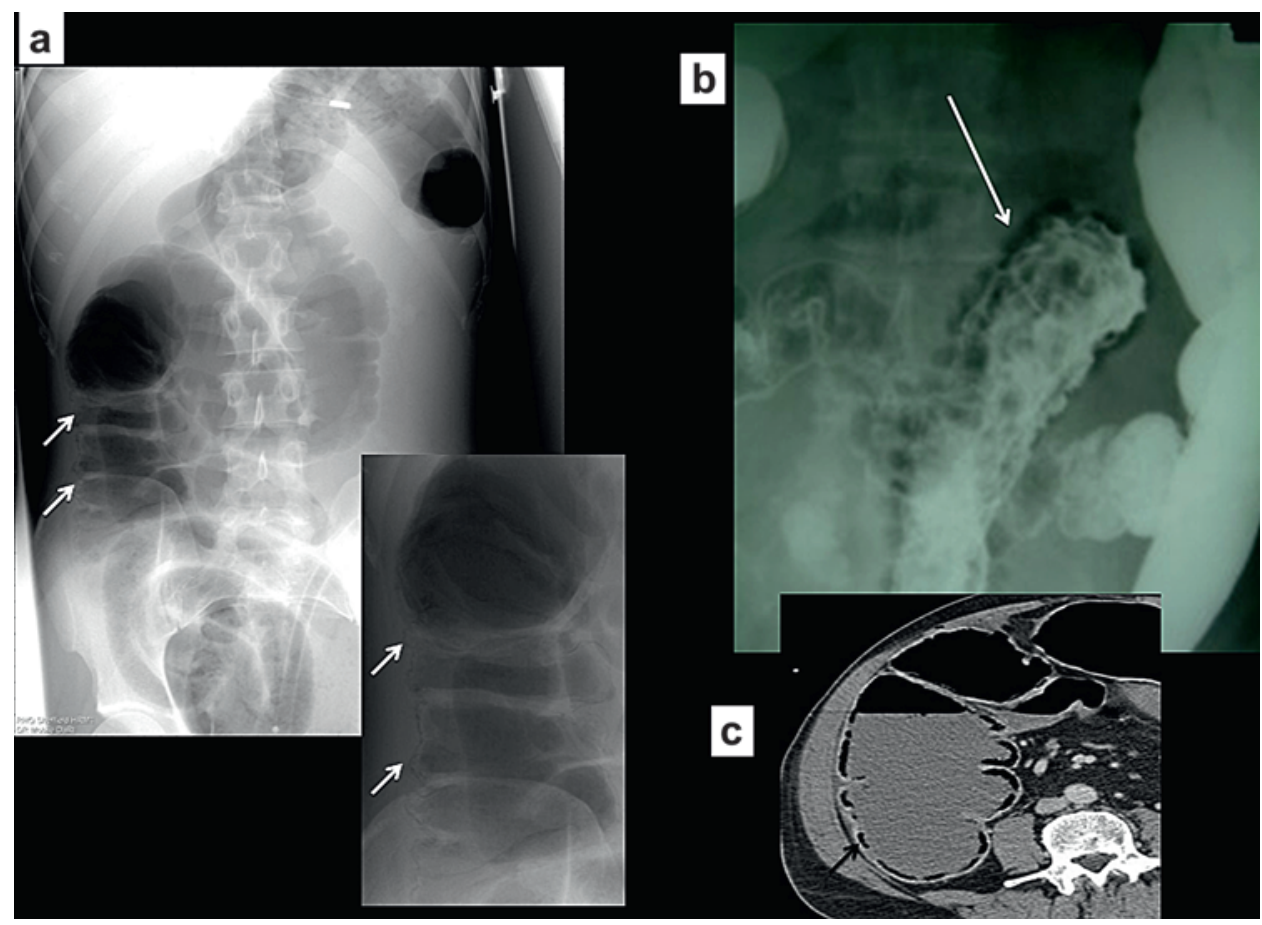


Figure 18 Intramural gas: emphysematous pyelonephritis. (A) Speckles of gas (white arrow) in the right flank on plain radiograph along with renal large calculi (black arrow). $(B, C) C T$ scan showing gas within the kidney due to emphysematous pyelonephritis in the context of renal stone disease.

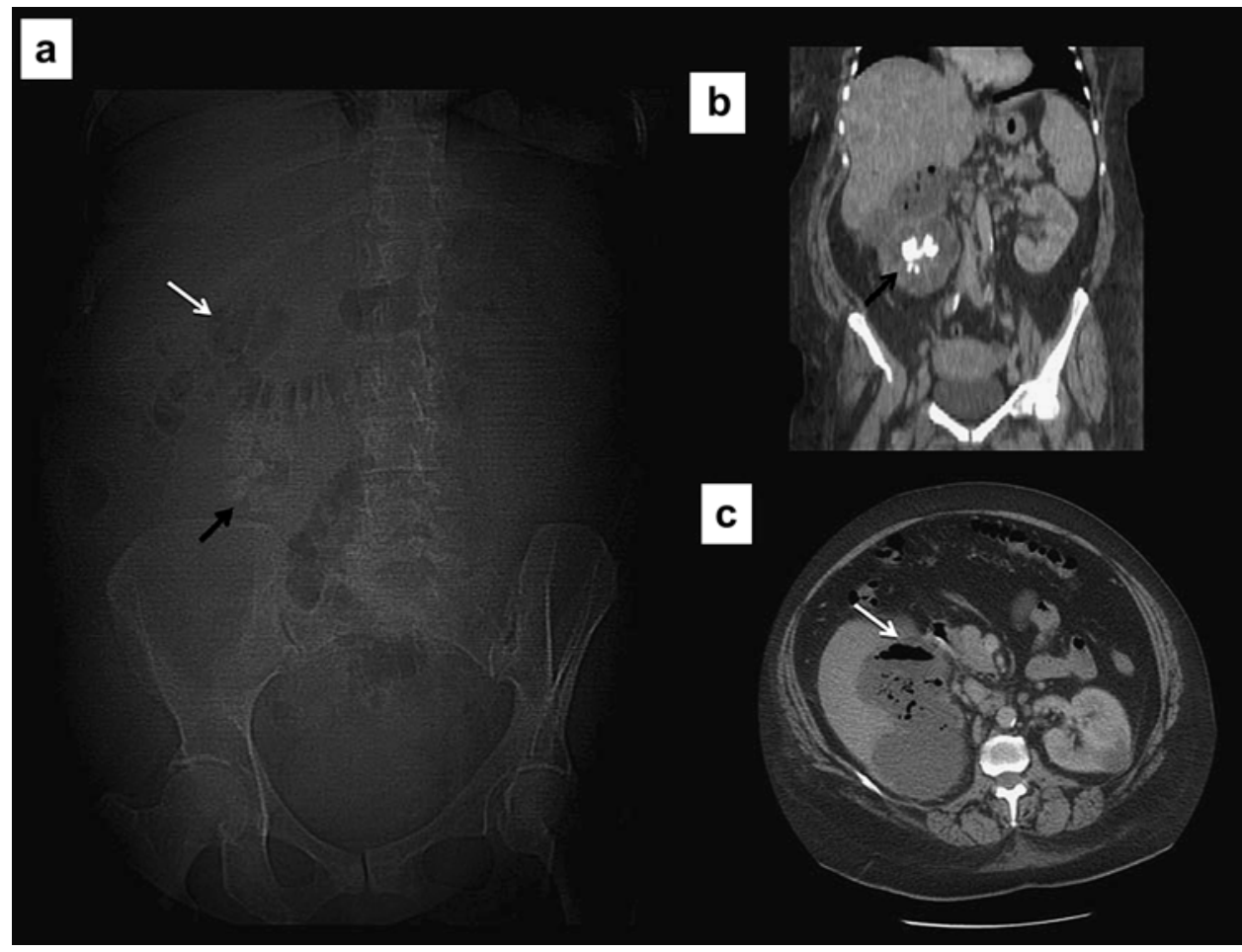

appendicitis, cholecystitis, and acute pancreatitis, which are readily detected by CT in over $95 \%$ of cases and over $80 \%$ in ultrasound, ${ }^{61013}$ with many other studies showing a similar high diagnostic accuracy of CT in the detection of the cause of acute abdominal pain. ${ }^{14}$ Many studies have previously shown that abdominal $\mathrm{x}$-rays are requested in inappropriate settings and make little difference in patient management ${ }^{15}$ and are not used in accordance with the Royal College of Radiologists guidance. ${ }^{16}$
Ultrasound does have a role in imaging of the acute abdomen. Accurate clinical assessment is paramount to direct the most appropriate modality of imaging. Ultrasound is excellent in the assessment of biliary disease, such as cholecystitis, renal disease and for abdominal collections. Small bowel obstruction and even free air may be detected on ultrasound often followed by further imaging with CT. One of the major benefits is the lack of ionising radiation. MRI has a long established role in the
Figure 19 Gas collections. $(A, B)$ Multiple locules of gas within the soft tissues and abdominal cavity. (C) Extensive subcutaneous gas secondary to necrotising fasciitis. (D) CT is useful for confirming the extent of the spread to the anterior abdominal wall and retroperitonium.

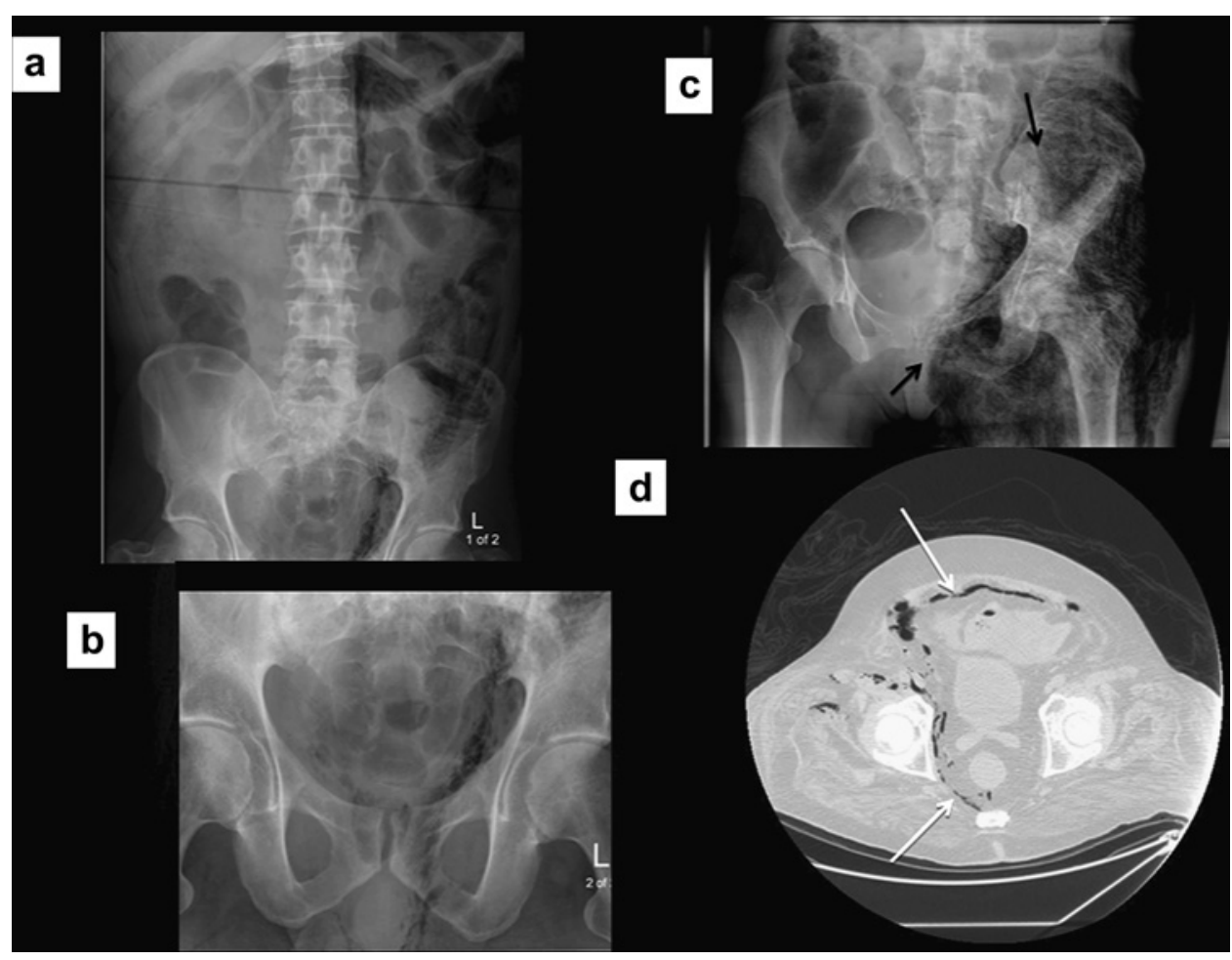


Figure 20 Deep intra-abdominal collections. (A) Plain radiograph showing a large left upper quadrant gasfilled collection (arrow). (B) Erect contrast swallow radiograph showing gas/fluid-filled left subphrenic collection impressing on the stomach (which contains contrast). (C) CT scan showing a large left subphrenic collection.

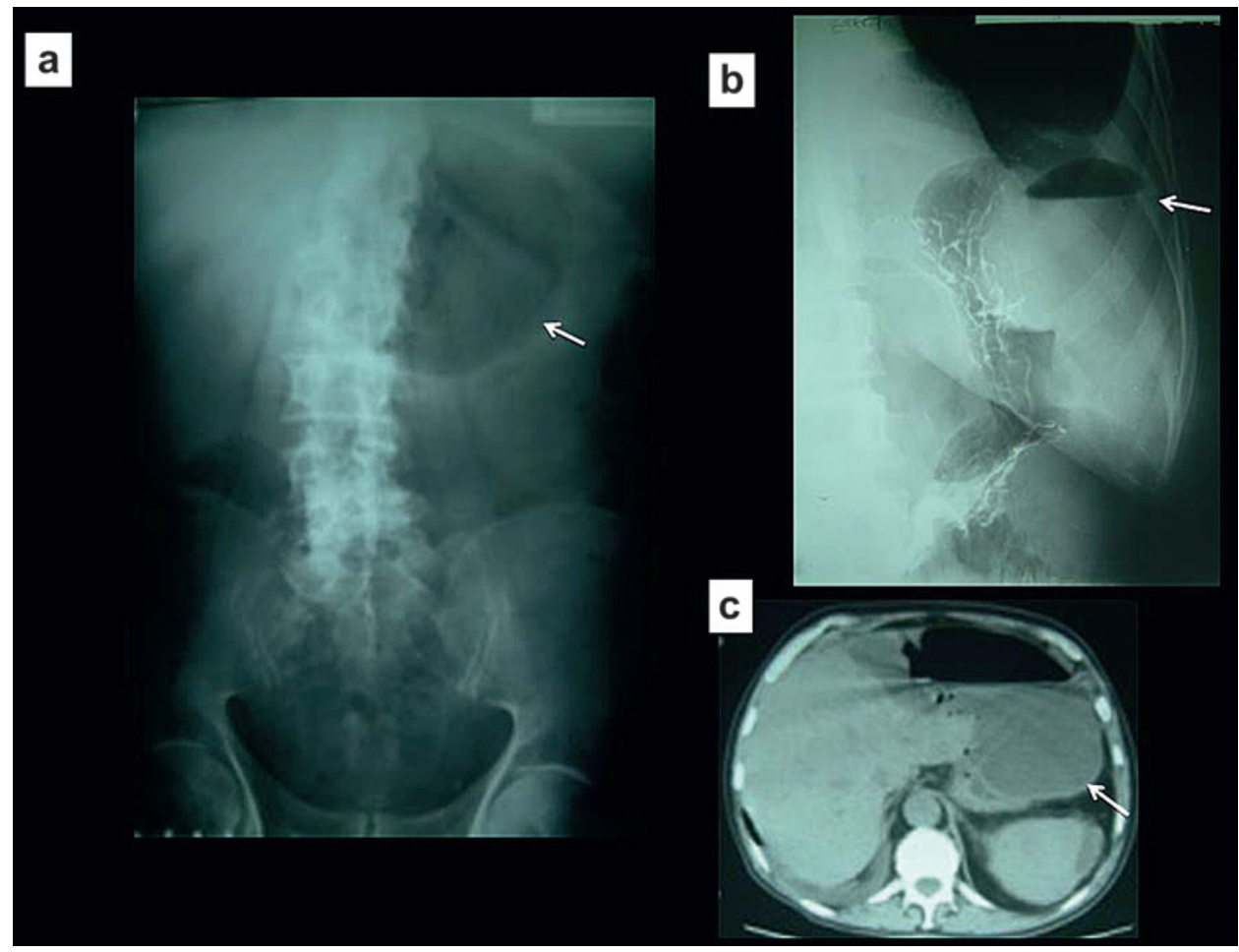

diagnosis of bile duct stones, but has also shown promising results in the diagnosis of appendicitis, particularly in pregnant women, ${ }^{17}$ and has been shown to be superior to CT in the diagnosis of cholecystitis. Currently, however, it has little place in the assessment of the acute abdomen in most institutions. ${ }^{18}$

As a result of its unrivalled accuracy, CT in the last 10 years has revolutionised the management of the acute abdomen. In addition to answering diagnostic dilemmas, it avoids negative laparotomies and directs the surgeon to the area of pathology, so surgery can be correctly planned.

\section{Current research questions}

Does the initial use of plain radiographs in the emergency setting actually change management?

- How does site of suspected obstruction compare on plain film and CT?

- What is the accuracy of MRI versus ultrasound in the evaluation of appendicitis?

\section{Key learning points}

- Abdominal radiographs in the setting of an acute abdomen are mainly useful for suspected bowel obstruction and suspected perforation.

- Abdominal radiographs have a much lower sensitivity than ultrasound and CT.

- The gas patterns depicted can be divided into intraluminal and extraluminal patterns.

- Plain abdominal radiographs may offer clues that should be examined for on every film to direct further management.

\section{Key references}

Stoker J, van Randen A, Lameris W, et al. Imaging patients with acute abdominal pain. Radiology 2009;253:31-46.

- Royal College of Radiologists. Making the Best Use of Clinical Radiology Services (MBUR). $6^{\text {th }}$ edn. Royal College of Radiologists, 2007. Available on online at http://mbur.nhs.uk/.

- Lameris W, van Randen A, van Es HW et al. Imaging strategies for detection of urgent conditions in patients with acute abdominal pain: diagnostic accuracy study. BMJ 2009;338:b2431

- Ahn S, Mayo-Smith W, Murphy B et al. Acute Nontraumatic abdominal pain in adult patients: abdominal radiography compared with CT evaluation. Radiology 2002;225:159-64.

- Maglinte D, Balthazar E, Kelvin F et al. The role of radiology in the diagnosis of small-bowel obstruction. AJR Am J Roentgenol 1997;168:1171-80.

\section{SUMMARY}

Abdominal films have an established place in the assessment of acute abdomen despite a low sensitivity. Accurate recognition of the gas patterns depicted, however, is important for prompt diagnosis and to direct further imaging, allowing definitive management.

\section{MULTIPLE-CHOICE QUESTIONS (TRUE (T)/FALSE (F); ANSWERS AFTER THE REFERENCES}

Intraluminal gas

1. Gas in the biliary tree extends to the periphery of the liver.

2. The classical features of air in the biliary tree, a calcified gallstone and small bowel obstruction are seen in approximately $80 \%$ of people with gallstone ileus.

3. Caecal volvulus usually occurs in the older population. 
4. Small bowel obstruction is most commonly due to hernia in the Western world.

5. The most common cause of large bowel obstruction is a colonic tumour.

Extraluminal gas

1. Emphysematous cholecystitis typically occurs in diabetics.

2. Extraluminal gas outlines the spleen in retroperitoneal perforation.

3. Pneumatosis coli requires urgent surgical intervention.

4. Rigler's sign is when air outlines the falciform ligament.

5. ERCP is a recognised cause of retroperitoneal perforation.

Competing interests None.

Provenance and peer review Commissioned; externally peer reviewed.

\section{REFERENCES}

1. Eisenberg RL. Clinical Imaging; An Atlas Of Differential Diagnosis. Lippincott Williams and Wilkins; 5th edn. Gastrointestinal Patterns, 2009:393.

2. Adam A, Dixon AK, Grainger RG, et al. Grainger and Allison's Diagnostic Radiology; A Text Book Of Medical Imaging. Churchill Livingstone, 5th edn, 2007:1:590-605.

3. Brant WE, Helms CA. Fundamentals Of Radiology. Lippincott Williams and Wilkins, 3rd edn, 2006:741-7.

4. Pontin AR, Barnes D, Joffe J, et al. Emphysematous pyelonephritis in diabetic patients. Br J Urol 1995;75:71-4.

5. Maglinte D, Balthazar E, Kelvin F, et al. The Role of radiology in the diagnosis of small-bowel obstruction. AJR Am J Roentgenol 1997:168:1171-80.

6. Ahn S, Mayo-Smith W, Murphy B, et al. Acute Nontraumatic abdominal pain in adult Patients: abdominal radiography compared with CT evaluation. Radiology 2002;225:159-64.

7. Atri M, McGregor C, Mclnnes M, et al. Multidetector helical CT in the evaluation of acute small bowel obstruction: comparison of non-enhanced (no oral, rectal or IV contrast) and IV enhanced CT. Eur J Radiol 2009:71:135-40.

8. Godfrey EM, Addley HC, Shaw AS. The use of computed tomography in the detection and characterisation of large bowel obstruction. N Z Med J 2009;122:57-73.

9. Oguro S, Funabiki T. Hosoda K, et al. 64-Slice multidetector computed tomography evaluation of gastrointestinal tract perforation site: detectability of direct findings in upper and lower GI tract. Eur Radiol 2010;20:1396-403.
10. Stoker J, van Randen A, Lameris W, et al. Imaging patients with acute abdomina pain. Radiology 2009;253:31-46

11. Tapakis JC, Thickman D. Diagnosis of pneumoperitoneum: abdominal CT vs. up- right chest film. J Comput Assist Tomogr 1992;16:713-16.

12. Hainaux $\mathbf{B}$, Agneessens $E$, Bertinotti $R$, et al. Accuracy of MDCT in predicting site of gastrointestinal tract perforation. AJR Am J Roentgenol 2006;187:1179-83.

13. Bhangu A, Richardson $\mathrm{C}$, Winer $\mathrm{H}$, et al. Value of initial radiological investigations in patients admitted to hospital with appendicitis, acute gallbladder disease or acute pancreatitis. Emerg Med J 2010:27:754-7.

14. Lameris W, van Randen A, van Es HW, et al. Imaging strategies for detection of urgent conditions in patients with acute abdominal pain: diagnostic accuracy study. BMJ 2009;338:b2431.

15. Feyler S, Williamson V, King D. Plain abdominal radiographs in acute medical emergencies: an abused investigation? Postgrad Med J 2002;78:94-6.

16. Making The Best Use Of Clinical Radiology Services (MBUR). 6th edn: Royal College of Radiologists, 2007.

17. Oto A. MR imaging evaluation of acute abdominal pain during pregnancy. Magn Reson Imaging Clin N Am 2006;14:489-501.

18. Stoker J. Magnetic resonance imaging and the acute abdomen. Br J Surg 2008;95:1193-4.

\section{ANSWERS}

Intraluminal gas

1. F-Gas in the biliary system is seen centrally over the liver. Gas in the portal veins extends to the periphery.

2. F-The classic triad is seen in less than $50 \%$ of cases

3. F-Caecal volvulus tends to occur in middle-aged adults. Sigmoid volvulus tends to occur in older people.

4. F-Adhesions are the most common cause in the Western world.

5. T.

Extraluminal gas

1. T-It is typically caused by gas-forming organisms

2. F-It outlines the retroperitoneal structures such as the psoas or kidney.

3. F-Pneumatosis coli is often an incidental finding.

4. F-Rigler's sign is air either side of the bowel wall.

5. T-The most common are duodenal or sigmoid perforations.

\section{Online Archive}

Visit our Online Archive - available back to 1925 . Subscribers may access the entire archive freely. Non-subscribers have free access to all articles prior to 2006. A simple one-time registration is required that grants access to all the free archive content, across all of our specialist titles. To view or to register visit pmj.bmj.com. 